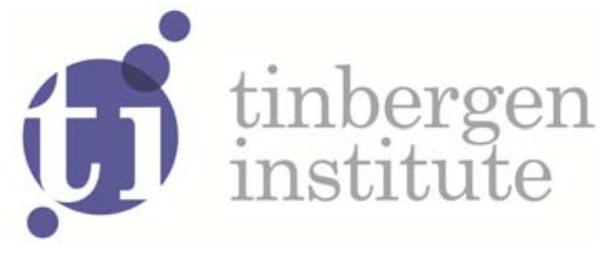

\title{
Predicting Covariance Matrices with Financial Conditions Indexes
}

\author{
Anne Opschoor \\ Dick van Dijk \\ Michel van der Wel
}


Tinbergen Institute is the graduate school and research institute in economics of Erasmus University Rotterdam, the University of Amsterdam and VU University Amsterdam.

More TI discussion papers can be downloaded at http://www.tinbergen.nl

Tinbergen Institute has two locations:

Tinbergen Institute Amsterdam

Gustav Mahlerplein 117

1082 MS Amsterdam

The Netherlands

Tel.: +31(0)205251600

Tinbergen Institute Rotterdam

Burg. Oudlaan 50

3062 PA Rotterdam

The Netherlands

Tel.: +31(0)10 4088900

Fax: $+31(0) 104089031$

Duisenberg school of finance is a collaboration of the Dutch financial sector and universities, with the ambition to support innovative research and offer top quality academic education in core areas of finance.

DSF research papers can be downloaded at: http://www.dsf.nl/

Duisenberg school of finance

Gustav Mahlerplein 117

1082 MS Amsterdam

The Netherlands

Tel.: +31(0)20 5258579 


\title{
Predicting Covariance Matrices with Financial
}

\section{Conditions Indexes}

\author{
Anne Opschoor* Dick van Dijk Michel van der Wel
}

This version: August 8, $2013^{\dagger}$

\begin{abstract}
We model the impact of financial conditions on asset market volatility and correlation. We propose extensions of (factor-)GARCH models for volatility and DCC models for correlation that allow for including indexes that measure financial conditions. In our empirical application we consider daily stock returns of US deposit banks during the period 1994-2011, and proxy financial conditions by the Bloomberg Financial Conditions Index (FCI) which comprises the money, bond, and equity markets. We find that worse financial conditions are associated with both higher volatility and higher average correlations between stock returns. Especially during crises the additional impact of the FCI indicator is considerable, with an increase in correlations by 0.15 . Moreover, including the FCI in volatility and correlation modeling improves Value-atRisk forecasts, particularly at short horizons.
\end{abstract}

Keywords: Dynamic correlations, Volatility modeling, Financial Conditions Indexes, Bank holding companies.

JEL: G17, G23, E44.

${ }^{*}$ Corresponding author, e-mail address: opschoor@ese.eur.nl. Postal address: Erasmus University Rotterdam, Erasmus School of Economics, P.O.Box 1738, 3000 DR, Rotterdam, The Netherlands. Phone number: +31(0)10-4088659.

$\dagger$ Anne Opschoor is from Erasmus University Rotterdam and the Tinbergen Institute. Van Dijk is from Erasmus University Rotterdam, Tinbergen Institute and ERIM. Van der Wel is from Erasmus University Rotterdam, CREATES, Tinbergen Institute and ERIM. We appreciate the comment of participants at the 19th International Conference on Computing in Economics and Finance (Vancouver, July 2013), the 33rd International Symposium on Forecasting (Seoul, June 2013), the 8th meeting of the Netherlands Econometric Study Group (NESG) (Amsterdam, June 2013), the 9th BMRC-QASS Conference on Macro and Financial Economics, (London, May 2013), the OMI-SoFIE Financial Econometrics Summer School (Oxford, August 2012) and seminars at the VU University Amsterdam and the Tinbergen Institute Rotterdam. Michel van der Wel is grateful to Netherlands Organisation for Scientific Research (NWO) for a Veni grant; and for support from CREATES, funded by the Danish National Research Foundation. We are responsible for all errors. 


\section{Introduction}

Volatilities and correlations of financial asset returns are crucial for many different purposes in financial management. Forming an investment portfolio using the mean-variance framework or related approaches requires the covariance matrix of the constituent assets returns, and so does calculation of Value-at-Risk for such portfolios. Pricing options depending on various underlying assets is highly sensitive to the correlations between their returns.

A key feature of volatilities and correlations of asset return volatilities is that they vary over time. Not surprisingly then, there is a substantial literature that attempts to link volatility and correlation dynamics to macroeconomic and financial fundamentals. For volatility most studies consider measures of aggregate market volatility in equity, bond and foreign exchange markets. Concentrating on the equity market, which is most relevant for this paper, it has been well documented that, in-sample, variables like bond returns, short term interest rates, producer prices and industrial production growth contain information for volatility (Schwert, 1989; Andersen et al., 2003, 2007, among many others). Out-ofsample, volatility forecasts can be improved by including short term interest rates (Glosten et al., 1993), and credit market variables including the commercial paper-to-Treasury spread, default returns, default spread and proxies for funding liquidity (Paye, 2012; Christiansen et al., 2012).

Most of the existing studies on the macroeconomic and financial drivers of return correlations focus on correlations between international equity returns or between stocks and bonds. International stock return correlations are positively associated with world market volatility and a trend; while they are negatively related to exchange rate volatility, term structure differentials across markets, real interest rate differentials, and the return on a world market index (Bracker and Koch, 1999). Bond and stock return correlations are only little explained by macro-economic fundamentals, but other factors, especially liquidity proxies, play a more important role (Baele et al., 2010; Christiansen and Ranaldo, 2007). Sheppard (2008) studies return correlations within an asset market, and using monthly covariances of the six Fama-French equity portfolio returns finds widespread evidence of statistically significant variation in conditional covariances attributable to financial and macro-economic state variables. 
In this paper, we study the impact of financial conditions on both volatility and correlations. This is motivated by a substantial body of recent evidence that particularly financial conditions are an important driver of the economy at large. Financial conditions impact monetary policy and macroeconomic variables, such as inflation and real GDP (see Goodhart and Hofmann, 2008; Guichard and Turner, 2008; Hatzius et al., 2010). In this literature, financial conditions are defined as the current state of financial variables that influence economic behavior and the future state of the economy (cf. Hatzius et al., 2010). From the literature on general economic conditions, it is known that high uncertainty causes an increase in volatility as investors react more strongly to new information (Pastor and Veronesi, 2008; Hamilton and Lin, 1996; Perez-Quiros and Timmermann, 2001), and asset returns may be more closely connected during bear markets than during bull markets (Longin and Solnik, 2001; Ang and Chen, 2002). So far little is known on how volatility and comovement of asset returns respond to changes in financial conditions. A notable exception is the work of Boudt et al. (2012), who document that financial conditions affect transition probabilities between volatility and correlation states. ${ }^{1}$

We propose several models to study the effects of financial conditions on asset volatilities and correlations in a multivariate GARCH framework. Specifically, we consider two approaches that both model the volatilities and correlations separately, which has become popular since the introduction of the DCC model of Engle (2002). Together, the models for volatility and correlation define the covariance matrix. The advantage of this set-up is that the effect of financial conditions on volatility and correlation can be disentangled. In the first approach, we extend the Spline-GARCH model of Engle and Rangel (2008) to include a measure of financial conditions as an explanatory variable. This model is combined with various specifications for the correlation dynamics. Specifically, we start with a simple linear relationship between (quasi) conditional correlations and a measure of financial conditions. This specification allows for flexibility in the correlation structure by allowing series specific impact of financial conditions. In addition, we adapt the cDCC model of Engle (2002) and Aelli (2013). In the second approach, we extend the Factor-Spline-GARCH model of Rangel and Engle (2012) by allowing financial conditions to affect the conditional variances of both the common market factor and the idiosyncratic returns. Indirectly, this also implies effects

\footnotetext{
${ }^{1}$ We turn to a more detailed comparison with our analysis later on in the introduction.
} 
of financial conditions on the correlations between asset returns.

We conduct an empirical analysis using daily equity returns of the largest US headquartered bank holding companies for the period January 1, 1994, through December 30, 2011. As a measure of financial conditions we use the Bloomberg Financial Condition Index (FCI), which is an equally weighted sum of money market indicators, bond market indicators and equity market indicators. ${ }^{2}$ Our analysis consists of two parts. First, we study the in-sample fit of the models. We compare model specifications with and without financial conditions, and test for significance of the impact of the FCI on both volatilities and correlations. Second, in an out-of-sample framework we consider whether Value-at-Risk predictions improve when financial conditions are included. The empirical application is inspired by Boudt et al. (2012), who use weekly returns of the same financial institutions to study the impact of the St. Louis Fed financial stress indicator on regime switching probabilities of a regime-switching GARCH model with two (equi-)correlation regimes. ${ }^{3}$

Our results show that financial conditions indeed do affect both the volatilities and the correlations of large US bank holding companies. Specifically, worse financial conditions are associated with higher volatilities and higher correlations. This result is both statistically and economically significant. For example, the FCI boosts the level of the correlation by 0.15 on average during the 2007-2008 crisis. In addition, Value-at-Risk (VaR) forecasts improve significantly compared to models without the FCI, such that less violations are made and the unconditional coverage match more closely to the nominal quantile. Finally, we find that our main result is not only driven by the VIX index - which is also captured by the Bloomberg FCI - but also by the other components of the Bloomberg FCI.

The remainder of the paper is organized as follows. Section 2 presents our modeling framework including our new proposed dynamic correlation model. In Section 3, we describe the data. Sections 4 and 5 discuss the in-sample and out-of-sample results, respectively.

\footnotetext{
${ }^{2}$ In recent years, several Financial Conditions Indexes (FCIs) (also sometimes called Financial Stress Indexes) have been developed, both by institutions including the IMF, World Bank and several branches of the Federal Reserve, as well as by major investment banks including Citi and Goldman Sachs. The various FCIs have in common that they summarize key indicators of financial market conditions into a single number. We opt for the Bloomberg FCI for two reasons: First, it is available at a daily frequency, and second, it comprises different financial markets.

${ }^{3}$ In contrast, we analyze daily returns and interact financial conditions directly with volatilities and (nonequi-)correlations. We use the Bloomberg FCI instead of the St. Louis Fed Indicator, since the Bloomberg index is available on a daily basis. We turn to data at the daily level as daily information has an important role for financial conditions during crises.
} 
Section 6 investigates the role of the VIX index in the Bloomberg FCI. Section 7 concludes.

\section{The modeling framework}

We use two different approaches to examine the role of financial conditions in shaping the volatilities and correlations of asset returns. First, we assume that volatilities can be described by a variation of the Spline-GARCH model of Engle and Rangel (2008) and combine this with variations of the dynamic conditional correlation (DCC) model of Engle (2002). Second, we extend the Factor-Spline-GARCH model of Rangel and Engle (2012), for volatilities and correlations simultaneously. In both cases we extend the models to incorporate the effects of financial conditions. We explain both modeling frameworks and the estimation of the models in the following subsections.

\subsection{The Spline-GARCH model for volatility with DCC for corre- lation}

Let $r_{i, t}$ denote the (excess) return for asset $i$ in period $t$. We consider the volatilities and correlations for $K$ assets, with their returns collected in the $K$-dimensional vector $r_{t} \equiv\left(r_{1, t}, \ldots, r_{K, t}\right)^{\prime}$, for $t=1, \ldots, T$. In our first modeling approach we assume that the conditional mean of $r_{t}$ is constant ${ }^{4}$, while its conditional covariance matrix is time-varying. That is, we have

$$
\begin{gathered}
\mathrm{E}_{t-1}\left[r_{t}\right]=\mu, \\
\mathrm{E}_{t-1}\left[\left(r_{t}-\mu\right)\left(r_{t}-\mu\right)^{\prime}\right]=H_{t},
\end{gathered}
$$

where $\mu=\left(\mu_{1}, \ldots, \mu_{K}\right)^{\prime}, \mathrm{E}_{t-1}$ denotes the expectation given the information set through time $t-1$, and $H_{t}$ the time-varying conditional covariance matrix. We disentangle the conditional correlations from the conditional variances by writing $H_{t}$ as

$$
H_{t}=D_{t}^{1 / 2} R_{t} D_{t}^{1 / 2}
$$

\footnotetext{
${ }^{4}$ The assumption of a fixed expected return is made purely for ease of exposition. It can be relaxed in a straightforward manner. In fact, in our empirical application we considered the possibility of timevarying expected returns by including autoregressive terms and possible explanatory variables such as the Bloomberg FCI in (1). All corresponding coefficients were insignificant, even at the $10 \%$ level.
} 
where $D_{t}=\operatorname{diag}\left(h_{i, t}\right), i=1, \ldots, K$ is a diagonal matrix with the conditional variances of the stock returns on the diagonal, and $R_{t}$ is the conditional correlation matrix.

We assume that the conditional variances can be described by a variant of the SplineGARCH model of Engle and Rangel (2008). In the standard Spline-GARCH model the volatility is decomposed into two components: a fast-moving GARCH term to capture typical high-frequency behavior, and a slow-moving spline function to capture more gradual movements due to, e.g., macroeconomic fluctuations. We adapt the model by replacing the second component with a function of a time-varying explanatory variable, such as a financial conditions index. The resulting model specification is given by

$$
\begin{aligned}
h_{i, t} & =g_{i, t} \tau_{i, t}, \\
g_{i, t} & =\omega_{i}+\alpha_{i} \frac{\left(r_{i, t-1}-\mu_{i}\right)^{2}}{\tau_{t-1}}+\gamma_{i} \frac{\left(r_{i, t-1}-\mu_{i}\right)^{2}}{\tau_{i, t-1}} I\left[\left(r_{i, t-1}-\mu_{i}\right)<0\right]+\delta_{i} g_{i, t-1}, \\
\tau_{i, t} & =\exp \left(\kappa_{i, 0}+\kappa_{i, 1} X_{t-1}\right) .
\end{aligned}
$$

with $g_{i, t}$ the fast-moving GARCH component and $h_{i, t}$ the slower-moving volatility component linked to the explanatory variable $X_{t-1}$, which is scaled to have mean zero. In the GARCH term $g_{i, t}$ in $(3), I[A]$ denotes an indicator function taking the value one when its argument $A$ holds true and zero otherwise. The corresponding term allows for different effects of positive and negative shocks on volatility, as in the GJR-model of Glosten, Jagannathan and Runkle (1993). For identification purposes, we impose the restriction $\omega_{i}=\left(1-\alpha_{i}-\delta_{i}-\gamma_{i} / 2\right)$, such that the unconditional mean of $g_{i, t}$ is equal to one. Accordingly, $g_{i, t}$ is referred to as the unit GARCH component. In the $\tau_{i, t}$ component the coefficient $\kappa_{i, 0}$ essentially is a scaling parameter determining the unconditional mean of $h_{i, t}$, while $\kappa_{i, 1}$ measures the impact of the exogenous variable $X_{t}$ on the volatility for asset $i$. We label this model the Spline-GARCH-X model, to highlight its dependence on the explanatory variable $X$. The main advantage of this approach over simply including the explanatory variable into a GARCH specification directly, is that the unit GARCH term $g_{i, t}$ in (3) does not change and that the variance $h_{i, t}$ is always positive because of the exponent term in $\tau_{i, t}$ in $(3)$.

In the spirit of the DCC model of Engle (2002), we model the correlation matrix $R_{t}$ in (2) by means of a quasi-correlation matrix $Q_{t}$. By appropriately scaling this quasi-correlation matrix the correlation matrix is obtained. This approach guarantees that the correlation 
matrix will have ones on the diagonal, and values between -1 and 1 in the off-diagonal elements. We start with a simple linear relationship between the quasi-correlation matrix $Q_{t} \equiv\left[q_{i j, t}\right], i, j=1, \ldots, K$ and the exogenous variable $X_{t}$ :

$$
Q_{t}=\bar{Q}+\gamma_{D C} X_{t-1} J
$$

with $J$ a $(K \times K)$ matrix of ones and $\bar{Q}$ the unconditional covariance matrix of the standardized returns $\varepsilon_{t}=D_{t}^{-1 / 2}\left(r_{i, t}-\mu_{i}\right)$, estimated by its sample analogue: $\bar{Q}=\frac{1}{T} \sum_{t=1}^{T} \varepsilon_{t} \varepsilon_{t}^{\prime}$. In this model the innovation in the correlations stems solely from the $X_{t-1}$ term. The impact of this explanatory variable is measured by $\gamma_{D C}$, and is equal across asset correlations. Finally, we scale the $Q_{t}$ matrix to ensure a valid time-varying conditional correlation matrix $R_{t}$ :

$$
R_{t}=Q_{t}^{*-1 / 2} Q_{t} Q_{t}^{*-1 / 2}
$$

We label this specification the DC-X model, for Dynamic Correlation with eXplanatory variables.

The specification in (4) may be considered restrictive, for the two properties just mentioned: the dynamics in the correlations are purely driven by the single variable $X_{t-1}$, while its impact is identical for all correlations. Both characteristics can be relaxed in a relatively straightforward manner. First, we may allow for a series specific impact of $X_{t}$ on the correlations by defining $\gamma_{D C}$ as a $(N \times 1)$ vector and specifying $Q_{t}$ as

$$
Q_{t}=\bar{Q}+\gamma_{D C} \gamma_{D C}^{\prime} X_{t-1}
$$

which resembles the generalized DCC [G-DCC] model of Cappiello et al. (2006) and Hafner and Franses (2009). Note that all elements of the $(K \times K)$ matrix $\gamma_{D C} \gamma_{D C}^{\prime}$ are positive, such that the direction of the effect of the $X_{t-1}$ variable is still the same across all correlations but its magnitude may obviously differ. We label this more flexible variation as the DC-X-F model.

Second, richer time-varying correlation patterns may be obtained by including the exogenous variable in the DCC model of Engle (2002). We start from the corrected DCC 
(cDCC) model proposed by Aelli (2013), given by

$$
Q_{t}=\bar{Q}+\alpha_{c D C C}\left(Q_{t-1}^{* 1 / 2} \varepsilon_{t-1} \varepsilon_{t-1}^{\prime} Q_{t-1}^{* 1 / 2}-\bar{Q}\right)+\beta_{c D C C}\left(Q_{t-1}-\bar{Q}\right)
$$

with $Q_{t}^{*}=\operatorname{diag}\left(q_{i i, t}\right), i=1, \ldots, K$ and $\alpha_{c D C C}$ and $\beta_{c D C C}$ scalar parameters. Following van Dijk et al. (2011), we extend this specification by replacing the unconditional (quasicorrelation matrix $\bar{Q}$ with a time-varying matrix $\bar{Q}_{t}$ driven by the explanatory variable $X$. This results in the model

$$
\begin{aligned}
Q_{t} & =\bar{Q}_{t}+\alpha_{c D C C}\left(Q_{t-1}^{* 1 / 2} \epsilon_{t-1} \epsilon_{t-1}^{\prime} Q_{t-1}^{* 1 / 2}-\bar{Q}_{t-1}\right)+\beta_{c D C C}\left(Q_{t-1}-\bar{Q}_{t-1}\right) \\
\bar{Q}_{t} & =\bar{Q}+\gamma_{c D C C} X_{t-1} J
\end{aligned}
$$

In this setting, the scalar coefficient $\gamma_{c D C C}$ has the same interpretation as in (4), namely the total effect of the variable $X_{t-1}$ on $Q_{t}$. We label this model as cDCC-X. ${ }^{5}$

\subsection{The Factor-Spline-GARCH model}

As a second approach to model volatilities and correlations and their relations with financial conditions, we adapt the Factor-Spline-GARCH model of Rangel and Engle (2012). This model extends the standard Spline-GARCH model in two dimensions. First, the asset returns are assumed to be adequately described by a one-factor version of the arbitrage pricing theory asset pricing model of Ross (1976). Second, time-varying volatilities and correlations of the asset returns are obtained jointly by specifying Spline-GARCH and DCC models for the market factor and the idiosyncratic returns. Specifically, the first extension of the Spline-GARCH model boils down to the assumption that the excess return of asset

\footnotetext{
${ }^{5} \mathrm{~A}$ natural question arises whether it is guaranteed that $Q_{t}$ is positive definite (pd) in our proposed models. In the standard cDCC model, $Q_{t}$ is positive definite when $\alpha_{c D C C} \geq 0, \beta_{c D C C} \geq 0$ and $\alpha_{c D C C}+$ $\beta_{c D C C} \leq 1$. When exogenous variables enter the specification these conditions are no longer sufficient. In case $\gamma_{c D C C} X_{t-1}$ is positive, $Q_{t}$ consists of the sum of a positive definite and a positive semi-definite matrix. As long as the effect of the latter matrix is not dominant, $Q_{t}$ is still positive definite. The same holds for the DC-X and DC-X-F models. In general it is however not guaranteed that $Q_{t}$ is positive definite. In the estimation procedure we use a pragmatic rule to ensure it is positive definite, through allowing $\gamma_{c D C C}$ to be negative, as long as the $Q_{t}$ matrix is positive definite for every $t$. In our empirical application this did not generate problems for both in the in-sample estimation of Section 4 and the forecasting exercise in Section 5 .
} 
$i, i=1, \ldots, K$ depends linearly on a single market factor:

$$
r_{i, t}=\xi_{i}+\beta_{i} r_{m, t}+u_{i, t}
$$

where $r_{m, t}$ denotes the market excess return, $\xi_{i}$ and $\beta_{i}$ are parameters to be estimated, and $u_{i, t}$ are the residuals or idiosyncratic returns. Allowing for cross-correlation between the idiosyncratic returns of different assets and for correlation between the market return and the residuals (that is, $\mathrm{E}_{t-1}\left[u_{i, t} u_{j t}\right] \neq 0$ for some $i \neq j$ and $\mathrm{E}_{t-1}\left[r_{m, t} u_{i, t}\right] \neq 0$ for some $i$ ), Rangel and Engle (2012) show that the conditional correlation between assets $i$ and $j$ at time $t$ is given by

$$
\begin{aligned}
\rho_{i j, t}= & \left(\beta_{j} \beta_{i} \mathrm{~V}_{t-1}\left[r_{m, t}\right]+\beta_{j} \mathrm{E}_{t-1}\left[r_{m, t} u_{i, t}\right]+\beta_{i} \mathrm{E}_{t-1}\left[r_{m, t} u_{j, t}\right]+\mathrm{E}_{t-1}\left[u_{i, t} u_{j, t}\right]\right) \\
& \times\left(\beta_{i}^{2} \mathrm{~V}_{t-1}\left(r_{m, t}\right)+\mathrm{V}_{t-1}\left[u_{i, t}\right]+2 \beta_{i} \mathrm{E}_{t-1}\left[r_{m, t} u_{i, t}\right]\right)^{-1 / 2} \\
& \times \quad\left(\beta_{j}^{2} V_{t-1}\left[r_{m, t}\right]+\mathrm{V}_{t-1}\left[u_{j, t}\right]+2 \beta_{j} \mathrm{E}_{t-1}\left[r_{m, t} u_{j, t}\right]\right)^{-1 / 2}
\end{aligned}
$$

where $\mathrm{V}_{t-1}$ denotes the variance given the information set through time $t-1$. Thus, as a result of the one-factor APT set-up of (9), the conditional correlation $\rho_{i j, t}$ is driven by various factors, including the variances of the market returns and the idiosyncratic returns, the $\beta$ 's, and the possible correlation between the market return and the idiosyncratic returns.

The idea of Rangel and Engle (2012) for the second departure of the spline-GARCH model is to now model the time-varying volatilities and correlations of the market factor, as well as and the $N$ idiosyncratic returns $u_{i, t}$. The Spline-GARCH model is used for all $(N+1)$ volatilities to discriminate between long- and short-term volatility effects. Here we adapt the Spline-GARCH model in a similar way as in the previous section, and replace the slow-moving spline by the explanatory variable $X_{t}$. This results in the following specification for the market return $r_{m, t}$ :

$$
\begin{aligned}
r_{m, t}= & \mu_{m}+\sqrt{g_{m, t} \tau_{m, t}} \varepsilon_{m, t}, \quad \text { where } \varepsilon_{m, t} \mid I_{t-1} \sim(0,1), \\
g_{m, t}= & \omega_{m}+\alpha_{m} \frac{\left(r_{m, t-1}-\alpha_{m}\right)^{2}}{\tau_{m, t-1}}+ \\
& \gamma_{m} \frac{\left(r_{m, t-1}-\mu_{m}\right)^{2}}{\tau_{m, t-1}} I\left[\left(r_{m, t-1}-\mu_{m}\right)<0\right]+\delta_{m} g_{m, t-1}, \\
\tau_{m, t}= & \exp \left(\kappa_{m, 0}+\kappa_{m, 1} X_{t-1}\right),
\end{aligned}
$$


with $\omega_{m}=\left(1-\alpha_{m}-\delta_{m}-\gamma_{m} / 2\right)$ for identification purposes. Likewise, the specification of the volatility of the idiosyncratic part of the returns reads:

$$
\begin{aligned}
u_{i, t}= & \sqrt{g_{i, t} \tau_{i, t}} \varepsilon_{i, t}, \quad \text { where } \varepsilon_{i, t} \mid I_{t-1} \sim(0,1), \\
g_{i, t}= & \omega_{i}+\alpha_{i} \frac{\left(r_{i, t-1}-\xi_{i}-\beta_{i} r_{m, t-1}\right)^{2}}{\tau_{i, t-1}}+ \\
& \quad \gamma_{i} \frac{\left(r_{i, t-1}-\xi_{i}-\beta_{i} r_{m, t-1}\right)^{2}}{\tau_{i, t-1}} I\left[\left(r_{i, t-1}-\xi_{i}-\beta_{i} r_{m, t-1}\right)<0\right]+\delta_{i} g_{i, t-1}, \\
\tau_{i, t}= & \exp \left(\kappa_{i, 0}+\kappa_{i, 1} X_{t-1}\right),
\end{aligned}
$$

with $\omega_{i}=\left(1-\alpha_{i}-\delta_{i}-\gamma_{i} / 2\right)$. Finally, we follow Rangel and Engle (2012) by assuming a cDCC process for the innovations of (11) and (12), collected as $\varepsilon_{t}^{t o t}=\left(\varepsilon_{t}^{m}, \varepsilon_{1, t}, \varepsilon_{2, t}, \ldots, \varepsilon_{K, t}\right)^{\prime}$ :

$$
Q_{t}=\left(1-\alpha_{c D C C}-\beta_{c D C C}\right) \bar{Q}+\alpha_{c D C C}\left(Q_{t-1}^{* 1 / 2} \varepsilon_{t-1}^{t o t} \varepsilon_{t-1}^{t o t^{\prime}} Q_{t-1}^{* 1 / 2}\right)+\beta_{c D C C} Q_{t-1},
$$

with $Q_{t}^{*}=\operatorname{diag}\left(q_{m m, t}, q_{11, t}, \ldots, q_{K K, t}\right)$ and scale the matrix $Q_{t}$ using (5). We label this model as Factor-Spline-GARCH model.

The main difference between the modeling approaches of the previous section and the one presented here, is that the first case volatilities and correlations of the equity returns are modeled directly through the Spline-GARCH-X model and the (c)D(C)C-X model, and also the impact of the exogenous variable $X_{t}$ is directly incorporated. In contrast, in the second case the link between $X_{t}$ and between volatilities and correlation is indirect. An increase of the explanatory variable in the market factor increases both the volatility and correlation of all assets, and likewise an increase of the explanatory variable in the idiosyncratic part increase both the volatility and relevant pair-wise correlations. An advantage of the FactorSpline-GARCH model is computational elegance: as the cDCC model for the remaining ideosyncratic terms $\varepsilon_{i, t}$ does not contain extra variables all matrix are by definition positive definite.

\subsection{Estimation}

This last subsection briefly discusses the estimation of the models put forward in the previous sections. For both modeling approaches, we follow the two-estimation procedure advocated by Engle (2002). In the first step, we estimate the parameters in the Spline-GARCH models 
for volatilities of the (idiosyncratic) returns of all $K$ assets separately, as well as for the market return in case of the Factor-Spline-GARCH model. ${ }^{6}$ Here we follow Rangel and Engle (2012) by assuming a conditional Student- $t$ distribution for the standardized returns. In the second step, we estimate the parameters of the correlation models using the standardized residuals from the Spline-GARCH volatility models. For the cDCC models we estimate these parameters by maximizing the (quasi) Composite Likelihood (CL) of Engle, Shephard and Sheppard (2008), as standard likelihood-based optimization implies biased estimates of $\alpha_{c D C C}$ for large $K$ (see Engle and Sheppard, 2005). The composite likelihood sums up likelihoods for each pair $(i, j)$ of assets:

$$
C L\left(\theta_{\text {corr }}\right)=\sum_{t=1}^{T} \sum_{i=1}^{K} \sum_{i>j} \log L_{i, j, t}
$$

where $\log L_{i, j, t}$ denotes the log-likelihood corresponding to the correlation part of asset $i$ and $j$ of the relevant correlation model and $\theta_{\text {corr }}$ stacks the correlation parameters. In practice, we follow the suggestion of Engle, Shephard and Sheppard (2008) and reduce the number of pairs by only considering randomly chosen contiguous pairs $i, i+1$. This does not affect the parameter estimates. We do not use the composite likelihood for the DC-X(-F) correlation models in (4) and (6), since the only innovation term is the $X_{t-1}$ variable. Using the regular likelihood in this case reduces the computational costs considerably.

For the Factor-Spline-GARCH model it is useful to note that estimation may be facilitated by first rewriting the factor setup as follows:

$$
r_{t}^{t o t}=\xi+B u_{t}^{t o t}
$$

where $r_{t}^{\text {tot }}=\left(r_{m, t}, r_{1, t}, r_{2, t}, \ldots, r_{K, t}\right)^{\prime}$ is the vector of excess returns at time $t, u_{t}^{\text {tot }}=\left(r_{m, t}, u_{1, t}, u_{2, t}, \ldots, u_{K, t}\right)^{\prime}$ such that it contains the market factor as well as the idiosyncratic returns, $B=\left(\begin{array}{cc}1 & 0_{1 \times N} \\ \beta & I_{N \times N}\end{array}\right)$, $\beta=\left(\beta_{1}, \beta_{2}, \ldots, \beta_{K}\right)^{\prime}$ and $\xi$ is a vector of intercepts. Then we have that $r_{t}^{t o t} \mid I_{t-1} \sim\left(\xi, H_{t}\right)$,

\footnotetext{
${ }^{6}$ For the Factor-Spline-GARCH model, we obviously first estimate the coefficients $\delta_{i}$ and $\beta_{i}$ in (9) by means of least squares.
} 
such that

$$
V_{t-1}=H_{t}=B D_{t} R_{t} D_{t} B^{\prime}
$$

with $D_{t}=\operatorname{diag}\left(\sqrt{\tau_{k, t} g_{k, t}}\right),(k=m, 1, \ldots, K), R_{t}$ the conditional correlation matrix as in $(5)$ with $Q_{t}$ denoted in (13). The corresponding correlation matrix of $r_{t}^{t o t}$ is then the covariance matrix scaled by the volatilities on its main diagonal: $\operatorname{diag}\left(H_{t}\right)^{-1 / 2} H_{t} \operatorname{diag}\left(H_{t}\right)^{-1 / 2}$.

\section{Data}

While financial conditions may affect the volatilities and correlations of equity returns of all kinds of firms, this should in particular be the case for financial institutions. For this reason we apply the models discussed in the previous section to returns on stocks of the largest US headquartered bank holding companies. We consider daily returns, over the period from January 1, 1994 to December 30, 2011. Following Boudt, Danielsson, Koopman and Lucas (2012), the banks in our sample are selected based on a ranking in terms of US domestic deposits constructed by the Federal Deposit Insurance Corporation. ${ }^{7}$ We select those banks that have been among the fifteen largest banks for at least three consecutive years during the sample period. Table 1 lists the 16 bank holding companies that satisfy this requirement and are included in our sample. The penultimate column in the table indicates the years for which the banks belonged to the top 15 of largest deposit holding companies in the US. Out of the 16 banks, six have been in the top list through the complete sample period, including Wells Fargo and Bank of America. Further, several banks have merged during the sample period (and some more than once), such as Nationsbanks Corp. and Chemical Banking Corp. We download daily price index returns from CRSP for all 16 banks. The last column provides the exact sample for which daily data is available. We do not have a balanced panel since the daily returns of Wachovia Corp. and National City Corp. are only available until December 30, 2008, as they merged in 2009 with Wells Fargo \& Co and PNC Financial Services, respectively.

Figure 1 provides an impression of the average level of the correlations between the

\footnotetext{
${ }^{7}$ http://www2.fdic.gov/sod/index.asp
} 
bank returns by showing the sample correlations for the period with an unbalanced sample (November 11, 1994 until December 30, 2011). The correlations of Wachovia Corp. and National City Corp. are based on the sample November 11, 1994, until December 30, 2008. The horizontal axis in the graph indicates the 16 banks (in the same order as in Table 1), and for each bank we plot the 15 sample correlations with the other banks. All correlations are positive and sizeable, ranging between 0.40 and 0.75 with an average close to 0.60 . The maximum correlation is 0.78 , between Wells Fargo \& Co. and National City Corp. It is also interesting to note that Capital One Financial Corp. consistently has the lowest correlation with each of the other bank holding companies.

The correlations between the different bank stock returns are not constant over time, as shown by Figure 2. This graph plots the average daily correlation of the Bank of America Corp. with the remaining 15 banks, as obtained by means of the Riskmetrics Model of Morgan (1994). ${ }^{8}$ We observe substantial time-variation in this average correlation. Most notably, it increases considerable to a level around 0.8 during the financial crisis (2007-2008), which is particularly pronounced compared to the substantial decline to around 0.1 at the end of 2006. A similar pattern occurs in 2011. The opposite pattern is observed in 2003-4, where the average correlation drops even to negative values after a period of high values around 0.8. For the other banks we find similar patterns.

In the models of Section 2 we extend volatility and correlation models with a financial conditions index. In our empirical application we use the Bloomberg FCI. This daily index tracks the overall conditions in the US money market, bond market and equity market. The FCI is an equal-weighted sum of three sub-indexes for each of these markets, see Rosenberg (2009). Table 2 shows the series of underlying indicators that form each sub-index, which are also equally-weighted. The underlying indicators as well as the FCI itself are presented as a Z-score, such that the index value should be interpreted as the number of standard deviations above or below its average, by construction over the period 1994-2008. Figure 3 shows the evolution of the Bloomberg FCI over time. The recent crisis in 2008 clearly stands out, but also other episodes of relatively low FCI values (below -2, say) can be associated with turmoil such as the Russian crisis and the LTCM bailout both in 1998, 9/11 in 2001,

\footnotetext{
${ }^{8}$ This model specifies the covariance matrix $\Sigma_{t}$ for day $t$ as $\Sigma_{t}=\lambda \Sigma_{t-1}+(1-\lambda) r_{t-1} r_{t-1}^{\prime}$. We use the default value of $\lambda=0.94$.
} 
Figure 1: Sample correlations of daily returns of financial institutions This figure depicts sample correlations of daily excess stock returns from 14 US bank holding companies from November 11, 1994 until December 30, 2011 (4312 observations) and correlations of Wachovia Corp. and National Citi Corp. (nr. 3 and 7) from November 11, 1994 until December 30, 2008 (3556 observations). Each column $i$ of small circles represents the sample correlations of stock $i$ with the 15 other constituents.

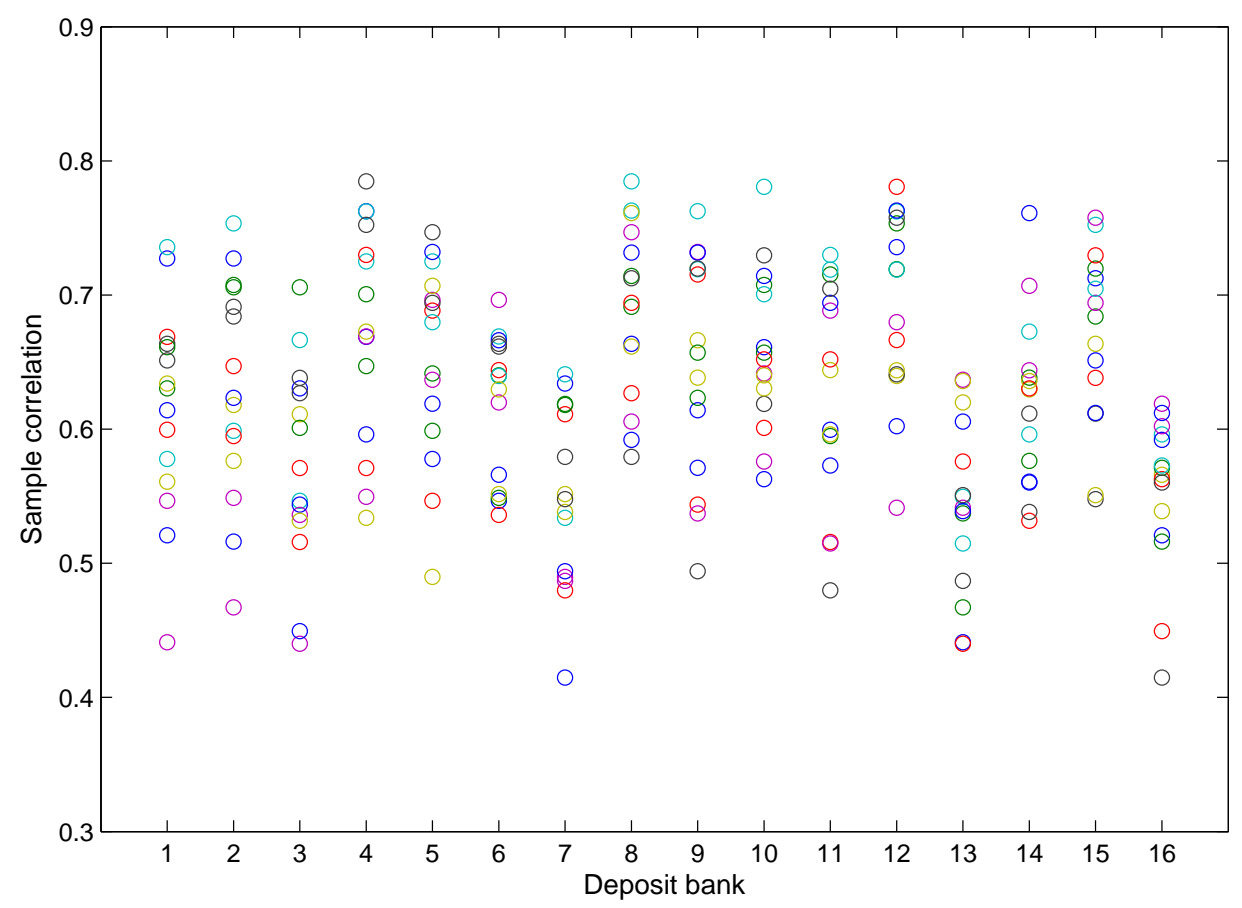


Figure 2: Average daily correlations of the Bank of America Corp. with other banks

This figure depicts average daily correlations between the Bank of America Corp. and the other US deposit bank returns from November 11, 1994 until December 30, 2011 (4312 observations), estimated with Riskmetrics. The correlations between the Bank of America Corp. and Wachovia Corp. and National City Corp. are based on the sample November 11, 1994 until December 30, 2008 (3556 observations).

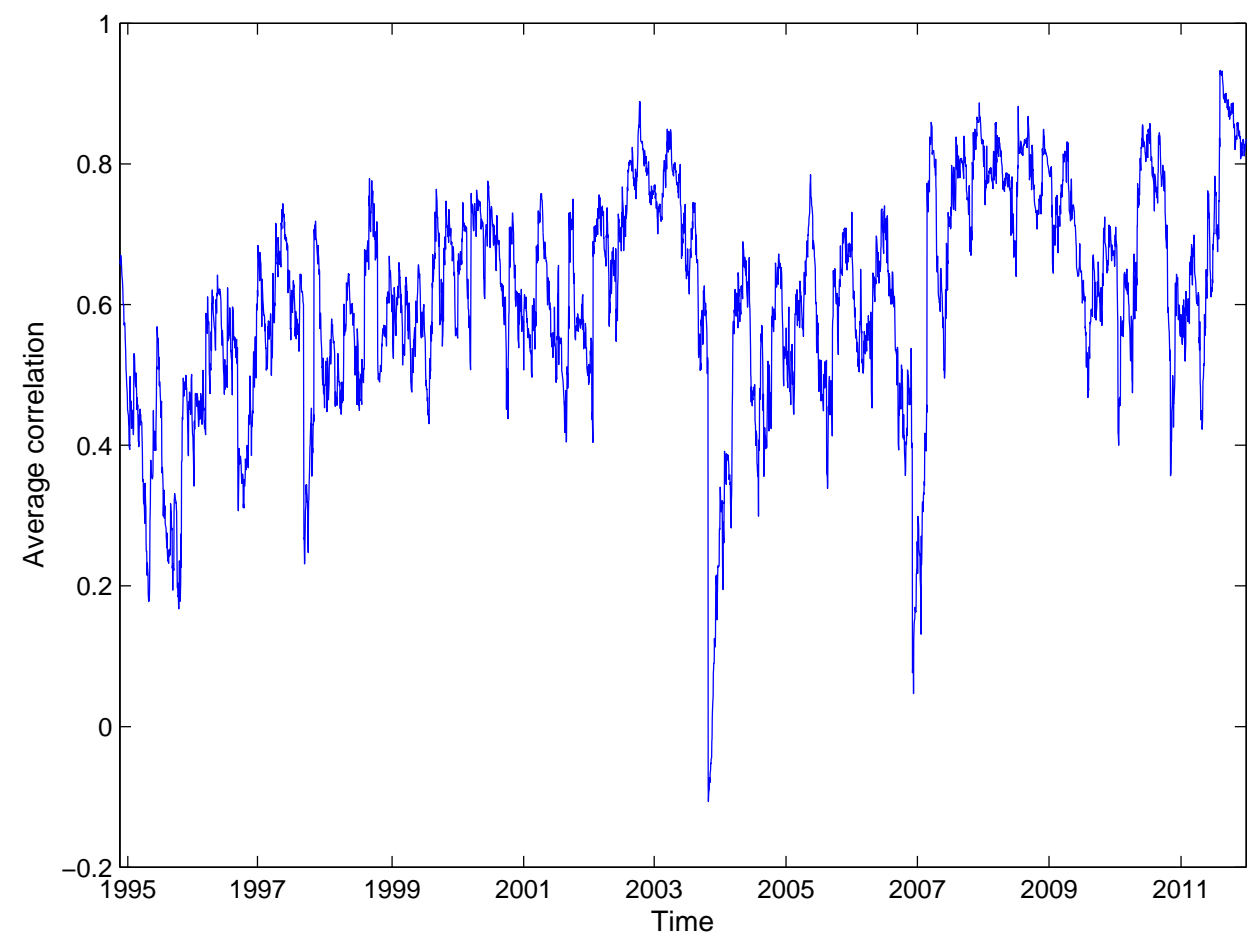


Table 1: Description of US bank holding companies

This table lists 16 US bank holding companies, together with the first and last year for which they belong to the top 15 of largest deposit banks in the US. Permno denotes the CRSP identifier. The table is modeled after Boudt, Danielsson, Koopman and Lucas (2012).

\begin{tabular}{|c|c|c|c|c|}
\hline & Permno & Name & In top 15 & Sample \\
\hline 1. & 34746 & Fifth Third Bancorp & $2001-2011$ & $1994-2011$ \\
\hline 2. & 35044 & Regions Financial Corp & $2005-2011$ & $1994-2011$ \\
\hline 3. & 36469 & First Union Corp, Wachovia Corp & $1994-2008$ & $1994-2008^{a}$ \\
\hline 4. & 38703 & Norwest Corp, Wells Fargo \& Co & $1994-2011$ & $1994-2011$ \\
\hline 5. & 47896 & Chemical Banking Corp, Chase Manhattan Corp, JP Morgan Chase \& Co & $1994-2011$ & $1994-2011$ \\
\hline 6. & 49656 & Bank of New York Mellon Corp & $2008-2011$ & $1994-2011$ \\
\hline 7. & 56232 & National City Corp & $1996-2008$ & $1994-2008^{b}$ \\
\hline 8. & 59408 & Nationsbank Corp, Bankamerica Corp, Bank of America Corp & $1994-2011$ & $1994-2011$ \\
\hline 9 . & 60442 & PNC Bank Corp, PNC Financial Services GRP Inc & $1994-2011$ & $1994-2011$ \\
\hline 10. & 64995 & Keycorp & $1994-2011$ & $1994-2011$ \\
\hline 11. & 66157 & US Bancorp & $1998-2011$ & $1994-2011$ \\
\hline 12. & 68144 & Suntrust Banks Inc & $1994-2011$ & $1994-2011$ \\
\hline 13. & 69032 & Morgan Stanley & $2009-2011$ & $1994-2011$ \\
\hline 14. & 70519 & Citigroup & $1999-2011$ & $1994-2011$ \\
\hline 15 . & 71563 & Southern National Corp NC, BB\&T Corp & $2000-2011$ & $1994-2011$ \\
\hline 16. & 81055 & Capital One Financial Corp & $2006-2011$ & $1995-2011$ \\
\hline
\end{tabular}

${ }^{a}$ Wells Fargo \& Co and Wachovia announced on October 3, 2008 they had agreed to merge. The purchase has been completed on December 31, 2008.

${ }^{b}$ PNC Financial Services announced October 24, 2008, its purchase of National City. 
the WorldCom bankruptcy in September 2002 and the Lehman collapse in 2008.

Table 2: Bloombergs' US FCI components and weights

This table lists the major sub-indexes and their underlying indicators that form the Bloomberg FCI. The table is modeled after Rosenberg (2009).

\begin{tabular}{llll} 
Category/item & Weight & \\
\hline Money Market & & \\
Ted Spread & $11.1 \%$ & \\
Commercial Paper/ T-Bill Spread & $11.1 \%$ & \\
Libor-OIS Spread Spread & $11.1 \%$ & + \\
\hline & \multicolumn{2}{l}{$33.3 \%$} & \\
\hline Bond Market & & \\
$\quad$ Baa/Treasury Spread & $6.7 \%$ & \\
Muni/Treasury Spread & $6.7 \%$ & \\
$\quad$ Swaps/Treasury Spread & $6.7 \%$ & \\
High Yield/Treasury Spread & $6.7 \%$ & \\
$\quad$ Agency/Treasury Spread & $6.7 \%$ & + & \\
\hline & & $33.3 \%$ & \\
\hline Equity Market & $16.7 \%$ & & \\
$\quad$ S\&P 500 Share Prices & $16.7 \%$ & + & \\
VIX Index & & $33.3 \%$ & + \\
\hline & & & $100 \%$ \\
\hline
\end{tabular}

\section{In-sample results}

This section analyzes in-sample estimation results of the volatility and correlation models, applied to daily deposit bank excess returns. The in-sample period goes from November 11, 1994, through December 30, 2011 (4,312 observations). We consider a balanced sample, such that we can also maximize the regular likelihood as discussed in Section 2.3, and therefore remove Wachovia Corp. and National City Corp (see Table 1).

We first turn to the results for the volatility models. Table 3 provides estimation results for the Spline-GARCH model of (3). To preserve space we report estimates of Morgan Stanley and Citigroup. See Table A.1 in Appendix B for results of the other banks. In Panel A we report estimates of the Spline-GARCH model with and without the FCI included as regressor. Specifically, the columns labeled (1) refer to the specification which does not include a financial conditions index, while the columns with label (2) add the Bloomberg FCI in the volatility specification. A key results from the table is that the coefficient on 


\section{Figure 3: The Bloomberg Financial Conditions Index}

This figure depicts the daily Bloomberg FCI from January 3, 1994, through December 30, 2011. The horizontal line denotes the sample mean of the indicator over the period 1994-2008. A positive value of the Bloomberg FCI indicates better (future) financial conditions of the US economy than on average while negative values means worsening of the financial conditions. The scale should be interpreted as the number of standard deviations above or below its average over the index 1994-2008.

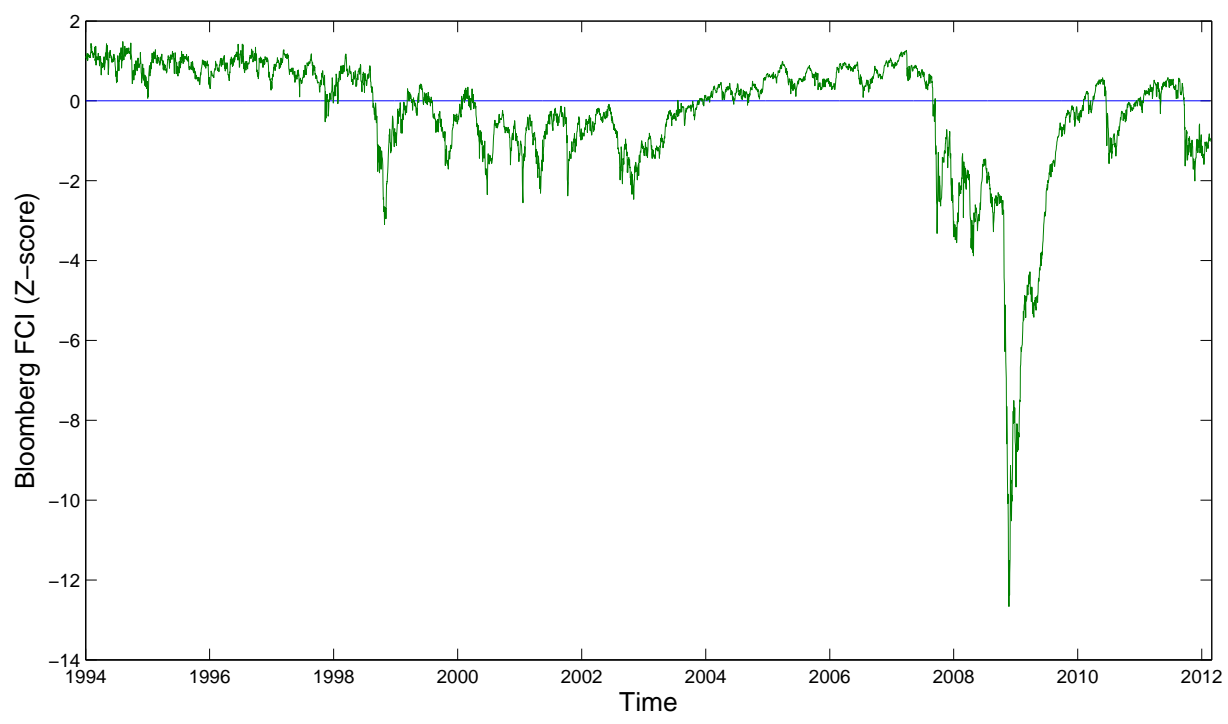


the FCI, $\kappa_{1}$, is significant at the $1 \%$ level. The coefficient is negative, thus a decrease in financial conditions causes volatility on the assets to increase. The effect is stronger for Morgan Stanley than for Citigroup. An decrease of the FCI by 1 point, increases Morgan Stanleys volatility by 1.05 percentage points. Further, the leverage effect, captured by the $\gamma$ parameter, reduces considerably for both banks when we include the Bloomberg FCI in the volatility specification. Hence a fraction of this effect is translated to economic fundamentals. Nevertheless, the $\alpha$ parameter indicates that the leverage effect is still stronger than the effect of the squared demeaned excess returns.

In Table 4 we report estimation results for the Factor Spline-GARCH model. In Panel A we report estimates of the volatility model of the ideosyncratic returns, as in (12), and in Panel B on the S\&P 500 market return from our second model approach, as in (11)). For comparability to Table 3 we focus on Morgan Stanley and Citigroup as well. The estimates of the remaining financial institutions are given in Table A.2 of Appendix B. The estimates of the idiosyncratic return as reported in Panel A are very similar to those reported in Panel A of Table 3. The impact of financial conditions on the volatility is somewhat weaker for the market factor (reported in Panel B), compared to the ideosyncratic return volatilities $(-0.32$ vs. -0.40$)$. Again, a part of the leverage effect translates to the FCI. Note that for the market return the leverages effect together with the FCI are the source of innovations for the volatility, while the squared demeaned excess market return does not influence the volatility.

Figures 4 and 5 depicts the in-sample volatilities of both banks for the period $1994-2011$ according to the two different model set-ups. The upper part of Figure 4 represents estimates of the square root of the unit GARCH term $g_{t}$ of the Spline-GARCH model without the FCI, while presented in (3), while the middle and the bottom part shows estimates of the decomposed volatility terms from the Spline-GARCH-X model with the Bloomberg FCI included as exogenous variable: the square root of the unit GARCH term $g_{t}$ (middle) and the square root of the "spline" component $\tau_{t}$ (bottom) containing the Bloomberg FCI. The figure indicates that including the Bloomberg FCI in the "spline" component $\tau_{t}$ results in less severe spikes of the unit GARCH term, as they are now incorporated in the $\tau$ component. For both banks the estimated volatility of the Spline-GARCH-X model is substantially higher during certain turmoil periods, especially in 1998 (Asia crisis) and the second half of 
2007 (credit crunch due to subprimes).

Figure 5 visualizes again in-sample volatilities of Morgan Stanley and Citigroup, but now corresponding with the factor Spline-GARCH approach from Section 2.2. In this figure the top part corresponds to the Factor-Spline-GARCH without the FCI included, and the bottom part to the Factor-Spline-GARCH model with the FCI included. In this case the differences between the volatility patterns is small. The reason could be due to the S\&P500 factor, which is now subtracted from the excess returns. At the same time, the S\&P 500 is also part of the Bloomberg FCI (see Table 2). Hence it could be that this factor dominates the other variables that are part of the Bloomberg FCI. Nevertheless, some peaks are higher, especially at the two aforementioned times (1998 and 2007).

The bottom part of Table 3 shows estimation results of alternative correlation models of Section 2.1, using daily returns of all 14 deposit banks. All models use standardized returns corresponding to specification where the volatilities are "corrected" for the impact of the FCI on volatility. For both the DC-X and cDCC-X correlation models, the FCI has a significant impact on the correlations between asset returns. A lower value of the FCI means worse financial conditions and increases correlations. This finding is in line with the literature (Longin and Solnik, 2001; Ang and Chen, 2002) that documents a stronger connection between asset returns during bear markets than during bull markets.

Figure 6 highlights the effect of including the FCI into correlation models by plotting the estimated correlation between Morgan Stanley and Citigroup. The three subgraphs correspond to the DC-X, cDCC, the cDCC-X and the Factor-Spline-GARCH model. As 'true correlation' is not observable, it is not possible to judge which of these correlation pattern is better. ${ }^{9}$ Nevertheless the plots highlight some interest in differences in the patterns, from which we draw three main conclusions. First, the DC-X model is less volatile than the Factor-Spline-GARCH and the $\mathrm{cDCC}(-\mathrm{X})$ models while during historical events like the 2007-2008 crisis the DC-X model produces a higher correlation than the remaining models. The average difference in correlation between the DC-X model and the cDCC(-X) model during the period October 1 through December 31, 2008, is equal to 0.15. All cDCC type of models shows a considerable drop in correlations during 1997, 2000, and 2009, while

\footnotetext{
${ }^{9}$ In the next section, we analyse the economic impact of the different correlation patterns by means of forecasting portfolio VaRs.
} 
Table 3: In-sample estimation results - Spline-GARCH Models

This table reports the estimation results of the $\mathrm{cDCC}(-\mathrm{X})$ and DC-X model. The volatility is formulated as a Spline-GARCH-X model:

$$
\begin{aligned}
& r_{i, t}=\mu_{i}+\sqrt{g_{i, t} \tau_{i, t}} \varepsilon_{i, t}, \\
& g_{i, t}=\left(1-\alpha_{i}-\delta_{i}-\gamma_{i} / 2\right)+\alpha_{i} \frac{\left(r_{i, t-1}-\mu_{i}\right)^{2}}{\tau_{i, t-1}}+\gamma_{i} \frac{\left(r_{i, t-1}-\mu_{i}\right)^{2}}{\tau_{i, t-1}} I\left[\left(r_{i, t-1}-\mu_{i}\right)<0\right]+\delta_{i} g_{i, t-1}, \\
& \tau_{i, t}=\exp \left(\kappa_{i, 0}+\kappa_{i, 1} F C I_{t-1}\right),
\end{aligned}
$$

and the correlation part is given by the $\mathrm{cDCC}(-\mathrm{X})$ or $\mathrm{DC}-\mathrm{X}(-\mathrm{F})$ model:

$$
\begin{aligned}
Q_{t} & =\bar{Q}+\gamma_{D C} F C I_{t-1} J, \quad(\mathrm{DC}-\mathrm{X}) \\
Q_{t} & =\bar{Q}_{t}+\alpha_{c D C C}\left(Q_{t-1}^{* 1 / 2} \epsilon_{t-1} \epsilon_{t-1}^{\prime} Q_{t-1}^{* 1 / 2}-\bar{Q}_{t-1}\right)+\beta_{c D C C}\left(Q_{t-1}-\bar{Q}_{t-1}\right), \quad(\mathrm{cDCC}-\mathrm{X}) \\
\bar{Q}_{t} & =\bar{Q}+\gamma_{c D C C} F C I_{t-1} J,
\end{aligned}
$$

with J a matrix of ones. We scale $Q_{t}$ from each model to obtain a valid correlation matrix:

$$
R_{t}=Q_{t}^{*-1 / 2} Q_{t} Q_{t}^{*-1 / 2},
$$

with $Q_{t}^{*}=\operatorname{diag}\left(q_{11, t}, \ldots, q_{K K, t}\right)$. Further, $r_{i, t}$ the daily excess return of holding bank $i,(i=1, \ldots, 14)$, and $F C I_{t}$ represents the Bloomberg FCI. The first part of the table shows Maximum Likelihood estimates of the

\begin{tabular}{|c|c|c|c|c|}
\hline \multicolumn{5}{|c|}{ Panel A: Volatility part } \\
\hline & \multicolumn{2}{|c|}{ Morgan Stanley } & \multicolumn{2}{|c|}{ Citigroup } \\
\hline Coeff. & (1) & (2) & (1) & $(2)$ \\
\hline$\mu$ & $0.029(0.027)$ & $0.021(0.030)$ & $0.015(0.019)$ & $0.006(0.033)$ \\
\hline$\alpha$ & $0.025(0.007)$ & $0.029(0.008)$ & $0.036(0.007)$ & $0.031(0.007)$ \\
\hline$\delta$ & $0.923(0.009)$ & $0.939(0.012)$ & $0.928(0.009)$ & $0.948(0.009)$ \\
\hline$\gamma$ & $0.094(0.014)$ & $0.046(0.014)$ & $0.069(0.012)$ & $0.038(0.014)$ \\
\hline$\kappa_{0}$ & $2.368(0.309)$ & $1.891(0.201)$ & $2.167(0.399)$ & $1.844(0.347)$ \\
\hline$\kappa_{1}$ & & $-0.403(0.050)$ & & $-0.323(0.089)$ \\
\hline$\nu$ & $7.203(0.667)$ & $7.460(0.759)$ & $7.151(0.608)$ & $7.072(0.613)$ \\
\hline $\log \mathrm{L}$ & -9900 & -9877 & -9228 & -9211 \\
\hline \multicolumn{5}{|c|}{ Panel B: Correlation part } \\
\hline Coeff. & DC-X & $\mathrm{cDCC}$ & cDCC-X & \\
\hline$\gamma_{D C}$ & $-0.214(0.005)$ & & & \\
\hline$\alpha_{c D C C}$ & & $0.026(0.001)$ & $0.019(0.001)$ & \\
\hline$\beta_{c D C C}$ & & $0.964(0.002)$ & $0.970(0.002)$ & \\
\hline$\gamma_{c D C C}$ & & & $-0.220(0.013)$ & \\
\hline $\log \mathrm{L}$ & 20835 & 20296 & 21023 & \\
\hline CL & & 11748 & 11904 & \\
\hline
\end{tabular}
Spline-GARCH(-X) model, assuming a conditional Student- $t$ distribution for $\varepsilon_{t}$. We show the volatilities corresponding to Morgan Stanley and Citigroup. Panel B shows estimation results of maximizing the Composite Likelihood (CL) and (quasi) Maximum Likelihood, using all 14 financial institutions. The LogL values of the DCC and cDCC-X models correspond with their regular likelihood values, obtained by plugging in the optimized CL estimates. Standard errors are in parentheses. The sample goes from November 11, 1994, through December 30, 2011 (4,312 observations). 
Table 4: In-sample estimation results - Factor-Spline-GARCH models

This table reports the estimation results of the Factor-Spline-GARCH model. The volatility is formulated as a Spline-GARCH-X model:

$$
\begin{aligned}
r_{i, t} & =\mu_{i, t}+\sqrt{g_{i, t} \tau_{i, t}} \varepsilon_{i, t}, \\
g_{i, t} & =\left(1-\alpha_{i}-\delta_{i}-\gamma_{i} / 2\right)+\alpha_{i} \frac{\left(r_{i, t-1}-\mu_{i, t}\right)^{2}}{\tau_{t-1}}+\gamma_{i} \frac{\left(r_{i, t-1}-\mu_{i, t}\right)^{2}}{\tau_{t-1}} I\left[\left(r_{i, t-1}-\mu_{i, t}\right)<0\right]+\delta_{i} g_{i, t-1}, \\
\tau_{i, t} & =\exp \left(\kappa_{i, 0}+\kappa_{i, 1} F C I_{t-1}\right),
\end{aligned}
$$

and the correlation part is given by the cDCC model:

$$
Q_{t}=\left(1-\alpha_{c D C C}-\beta_{c D C C}\right) \bar{Q}+\alpha_{c D C C}\left(Q_{t-1}^{* 1 / 2} \epsilon_{t-1} \epsilon_{t-1}^{\prime} Q_{t-1}^{* 1 / 2}\right)+\beta_{c D C C} Q_{t-1},
$$

where we scale $Q_{t}$ from each model to obtain a valid correlation matrix:

$$
R_{t}=Q_{t}^{*-1 / 2} Q_{t} Q_{t}^{*-1 / 2},
$$

with $Q_{t}^{*}=\operatorname{diag}\left(q_{11, t}, \ldots, q_{K K, t}\right)$. Further, $r_{i, t}$ the daily excess return of holding bank $i,(i=m, 1, \ldots, 16)$ and the S\&P 500 market return. In addition, $\mu_{i, t}=\xi_{i}+\beta_{i} r_{m, t}$ with $r_{m, t}$ the excess market return at day $t$. Otherwise, $\mu_{i, t}=\mu_{m}$ in case of the market return. $F C I_{t}$ represents the Bloomberg FCI and $Q_{t}^{*}=\operatorname{diag}\left(q_{m m, t}, q_{11, t}, \ldots, q_{K K, t}\right)$. The first part of the table shows Maximum Likelihood estimates of the Spline-GARCH(-X) model, assuming a conditional Student- $t$ distribution for $\varepsilon_{t}$. We show the volatilities corresponding to Morgan Stanley and Citigroup. Panel B shows similar results, but for the daily S\&P500 returns. Panel $\mathrm{C}$ shows estimation results of maximizing the Composite Likelihood (CL) using the standardized returns of all assets including the market return. The LogL value of the cDCC model corresponds with the regular likelihood, obtained by plugging in the optimized CL estimates. Standard errors are in parentheses. The sample goes from November 11, 1994 through December 30, 2011 (4312 observations).

\begin{tabular}{lcccc}
\hline \multicolumn{2}{l}{ Panel A: Ideosyncratic Volatility } \\
\hline \multicolumn{4}{c}{ Morgan Stanley } & \multicolumn{3}{c}{ Citigroup } \\
\hline Coeff. & $(1)$ & $(2)$ & $(1)$ & $(2)$ \\
\hline$\beta$ & $1.605(0.023)$ & $1.612(0.023)$ & $1.301(0.020)$ & $1.306(0.020)$ \\
$\alpha$ & $0.028(0.007)$ & $0.018(0.005)$ & $0.065(0.012)$ & $0.054(0.012)$ \\
$\delta$ & $0.943(0.009)$ & $0.976(0.007)$ & $0.914(0.012)$ & $0.929(0.013)$ \\
$\gamma$ & $0.050(0.011)$ & $0.010(0.009)$ & $0.036(0.014)$ & $0.026(0.012)$ \\
$\kappa_{0}$ & $1.649(0.315)$ & $1.203(0.278)$ & $1.588(0.442)$ & $1.241(0.361)$ \\
$\kappa_{1}$ & & $-0.401(0.039)$ & & $-0.401(0.053)$ \\
$\nu$ & $5.537(0.393)$ & $5.918(0.453)$ & $5.987(0.445)$ & $6.154(0.485)$ \\
\hline LogL & -8362 & -8327 & -7739 & -7715 \\
\hline
\end{tabular}

Panel B: Market Volatility

\begin{tabular}{lcr}
\hline Coeff. & $(1)$ & \multicolumn{1}{c}{$(2)$} \\
\hline$\mu$ & $0.042(0.012)$ & $0.036(0.013)$ \\
$\alpha$ & $0.000(0.009)$ & $0.000(0.008)$ \\
$\delta$ & $0.923(0.012)$ & $0.924(0.012)$ \\
$\gamma$ & $0.133(0.017)$ & $0.114(0.017)$ \\
$\kappa_{0}$ & $0.037(0.336)$ & $-0.101(0.112)$ \\
$\kappa_{1}$ & $-0.316(0.064)$ \\
$\nu$ & $9.194(1.144)$ & $9.546(1.293)$ \\
\hline LogL & -6126 & -6116 \\
\cline { 1 - 1 } \\
Panel C: Correlations \\
\cline { 1 - 2 } Coeff. & cDCC \\
\cline { 1 - 2 }$\alpha_{c D C C}$ & $0.010(0.001)$ \\
$\beta_{c D C C}$ & $0.987(0.001)$ \\
\hline LogL & 8478 \\
CL & 4051 \\
\hline
\end{tabular}




\section{Figure 4: In-sample volatilities - Spline-GARCH models}

This figure depicts daily estimated volatilities of Morgan Stanley and Citigroup from November 11, 1994, through December 30, 2011. The top part denotes estimates of the square root of the unit GARCH term $g_{t}$ from the Spline-GARCH model without exogenous variables included. The middle and bottom part shows estimates of the GARCH term $\sqrt{g_{t}}$ (middle part) and the evolution of the 'spline' component $\sqrt{\tau_{t}}$ (bottom part) from the Spline-GARCH-X model with the Bloomberg FCI included as exogenous variable.

Morgan Stanley
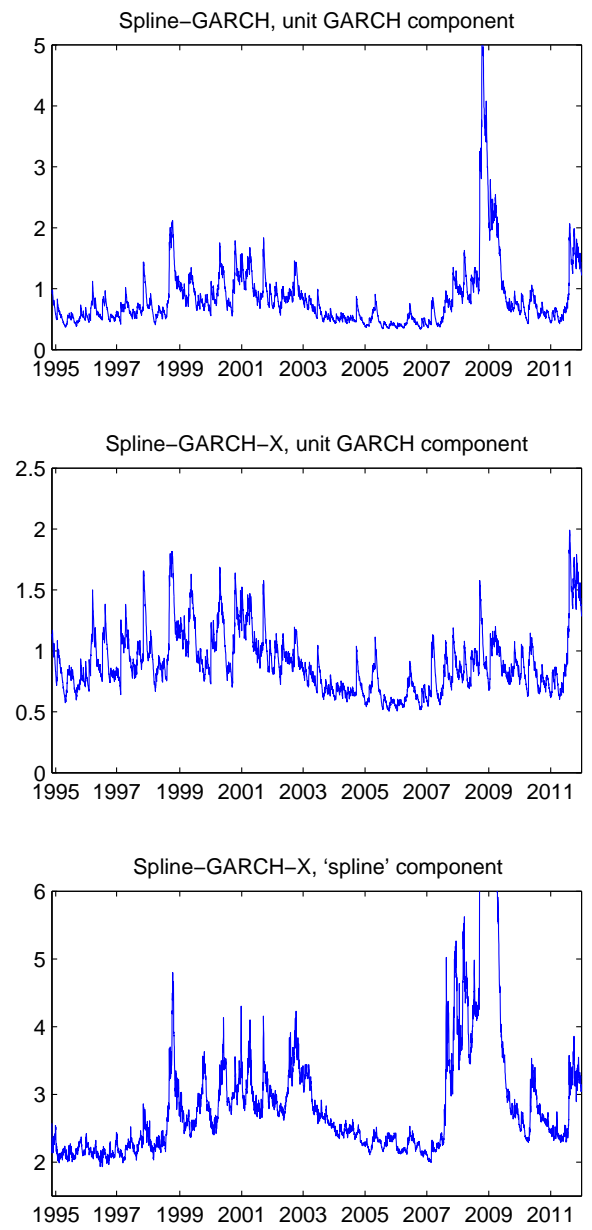

Citigroup
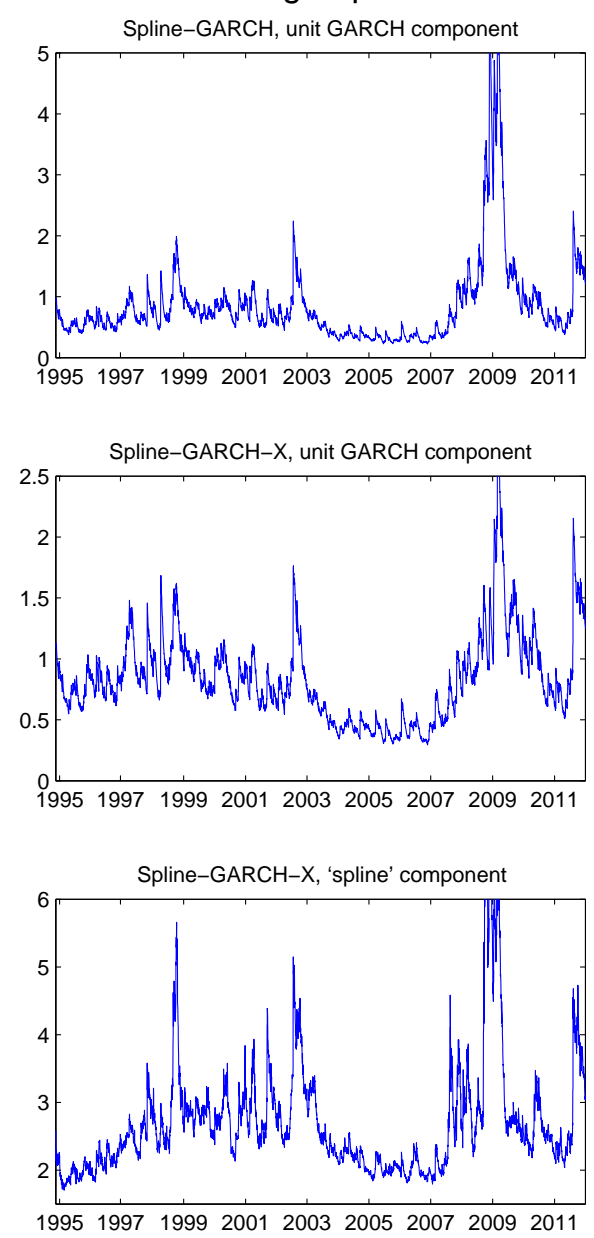
Figure 5: In-sample volatilities - Factor-Spline-GARCH models

This figure depicts daily estimated volatilities of Morgan Stanley and Citigroup from November 11, 1994, through December 30, 2011. The top part denotes estimates of the conditional volatility $\sqrt{h_{t}}$ from the Factor-Spline-GARCH model without exogenous variables included. The bottom part shows estimates of conditional volatility from the Factor-Spline-GARCH model with the Bloomberg FCI included as exogenous variable.
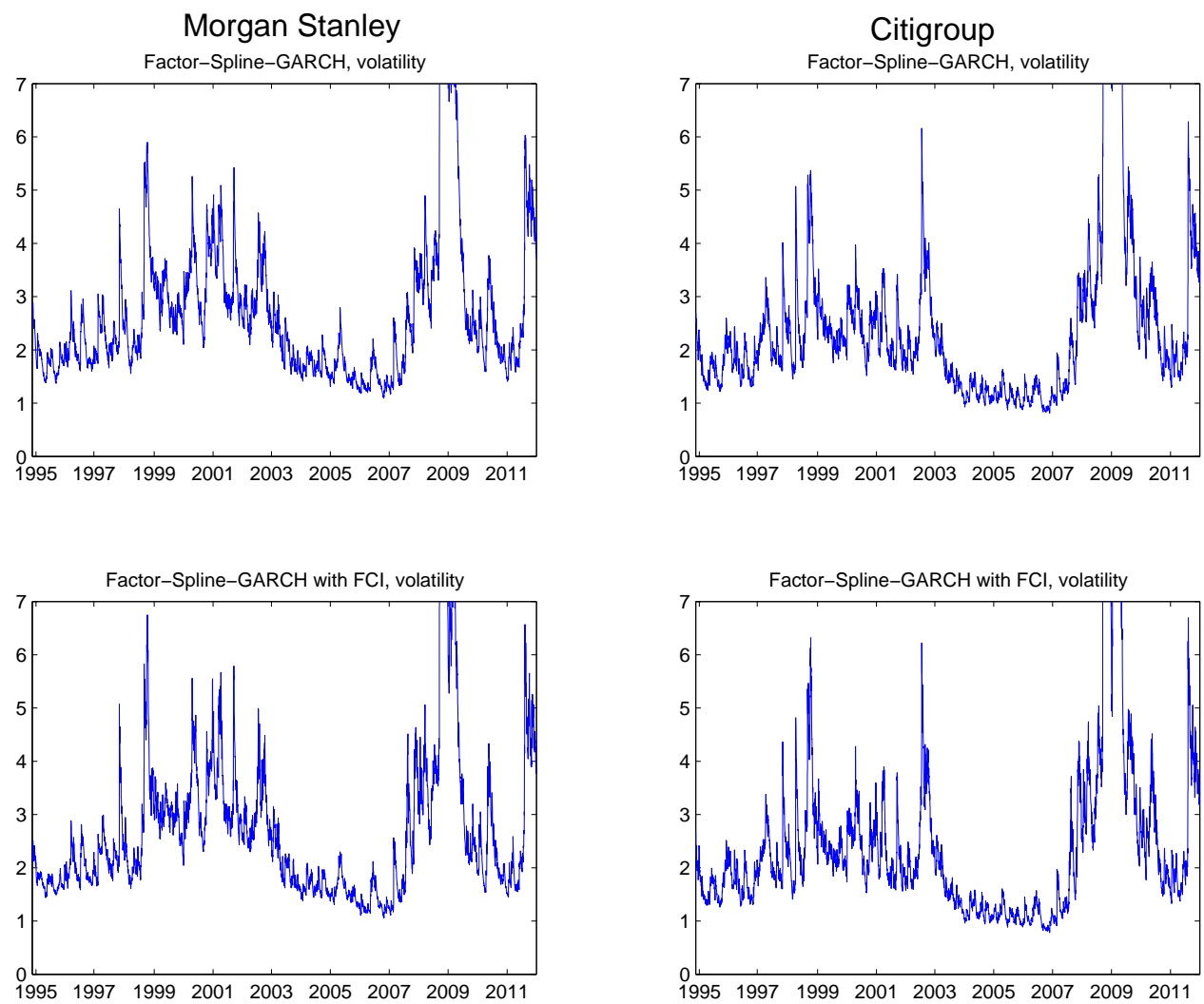
the DC-X model does not. A second finding is the similarity between the Factor-SplineGARCH correlations and the $\operatorname{cDCC}(-\mathrm{X})$ correlations, although the former is more volatile and exhibits more spikes. Hence it seems the one-factor approach with cDCC correlations for the ideosyncrasic returns captures the same dynamics as the cDCC-X correlations for the excess returns. Third, the inclusion of a financial conditions index in the cDCC model or in the Factor-Spline-GARCH model does play a role, although the effect is more visible in the cDCC model than in the Factor-Spline-GARCH model. Similar as in the volatilities, it seems that the market return factor captures a part of the effect of the Bloomberg FCI.

In summary, the results suggest that the Bloomberg FCI has a statistically significant impact on both volatilities and correlations of bank returns. Lower financial conditions will increase the volatilities of bank returns as well as the correlation between financial institutions, in particular during crises periods.

\section{$5 \quad$ Forecasting portfolio Value-at-Risk}

We assess the economic value of including the Bloomberg FCI into correlations and volatilities by considering portfolio Value-at-Risk forecasts. The VaR is a particular quantile of the conditional portfolio distribution which is of interest for risk-management in general. Based on this number, financial institutions for example have to decide how much capital should be hold for possible losses.

In the context of our models presented in section 2, we have assumed a particular specification for the conditional mean and variance. It follows then that the $q \%$ portfolio $\mathrm{VaR}$ at time $t$ can be expressed as

$$
V a R_{t}^{q}=\mu_{P, t}+z_{q} \sqrt{w_{t}^{\prime} H_{t} w_{t}}
$$

with $\mu_{P, t}$ the portfolio conditional mean, $w_{t}$ the portfolio weights, and $H_{t}$ the (forecasted) portfolio covariance matrix such that $\sqrt{w_{t}^{\prime} H_{t} w_{t}}$ denotes the portfolio standard deviation at time $t$. Finally $z_{q}$ represents the q-th quantile of the standardized portfolio distribution.

In the analysis, we consider a equally-weighted portfolio, such that $w_{t}=w=1 / K$, with $K$ the number of assets. In addition, $\mu_{P, t}=\mu_{P}=\mu^{\prime} w$ with $\mu$ defined in (1) in case of the 
Figure 6: In-sample correlations

This figure depicts the estimated correlation between daily excess returns of Morgan Stanley and Citigroup from November 11, 1994, through December 30, 2011 (4,312 observations) according to five different correlation models. The upper part shows in-sample correlations from the cDCC (blue line) and the DC-X model of (4) (red line), while the middle corresponds with estimated correlations of again the cDCC model (blue line) and the cDCC-X model (red line). The bottom part provides the estimated correlations of the Factor-Spline-GARCH model (9)-(12) with (blue line) and without (red line) including the Bloomberg FCI.
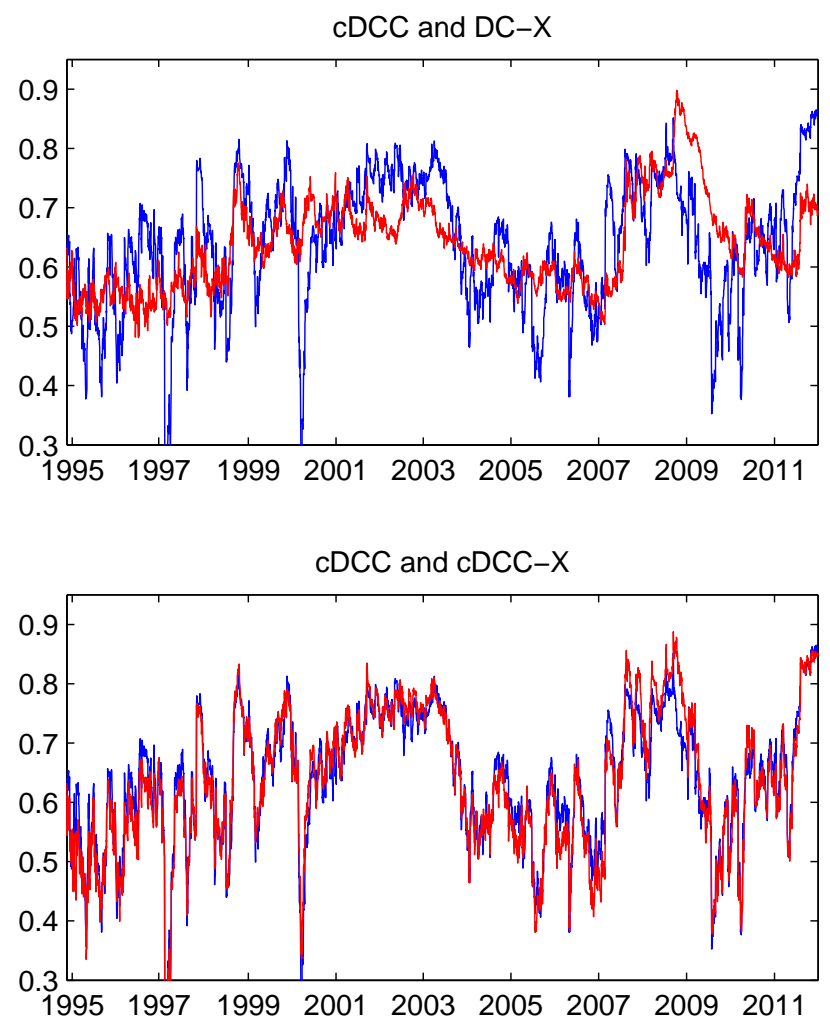

Factor-Spline-GARCH and Factor-Spline-GARCH with FCI

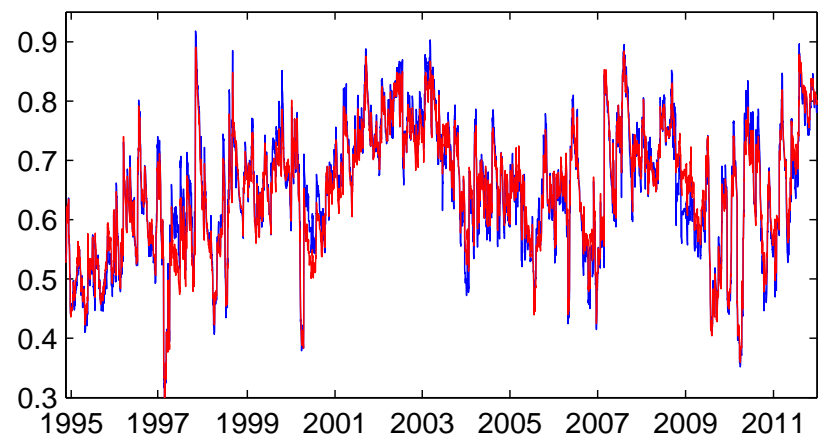


cDCC-X and DC-X models and $\mu=\alpha+\alpha_{m} \beta$ in case of the Factor-Spline-GARCH model (see (9)-(13)). Further, $H_{t}$ is the predicted $h$-step ahead covariance matrix, based on the Factor-Spline-GARCH (with and without the FCI included), the cDCC(-X) and the DC$\mathrm{X}$ models. We use an expanding-window to estimate the volatility/correlation parameters, starting with a sample of 1500 observations, which corresponds to the years $1995-2000 .{ }^{10}$ We re-estimate the parameters repeatedly after each 50 observations, which corresponds roughly to two months. At each point in time, we compute a 1\%, 5\% and 10\% 1-day portfolio VaR. In addition, we consider 5\% and 10\% 10-day portfolio VaRs and estimate a suitable ARMA process to forecast the Bloomberg FCI 10 steps ahead. ${ }^{11}$ Finally, we create 10 different sub-series in order to circumvent possible serial correlation in the 10-day portfolio VaRs. Hence sub-series $j$ contains the forecasts $\left\{V a R_{j}^{q}, V a R_{j+10}^{q}, V a R_{j+20}^{q}, \ldots\right\}$ for $j=1, \ldots, 10$.

Let us continue by focusing on the quantile $z_{q}$ in (17) of the standardized portfolio returns. We emphasize the importance of this number as it has a direct impact on the VaR. Given the non-normality of our financial institution excess returns, the use of the traditional values corresponding with the standard-normal distribution is not desirable. We therefore estimate $z_{q}$ using two different approaches. The first approach considers the empirical quantiles corresponding with the in-sample standardized portfolio returns which is most reliable as one does not make a parametric judgement about the distribution. The second approach estimates a Student- $t$ distribution on the in-sample standardized portfolio returns such that $z_{q}=t_{\hat{\nu}_{P}}^{-1}(q)$. By doing so, we avoid a computationally involved estimation of our multivariate models assuming a conditional Multivariate Student- $t$ distribution. ${ }^{12}$

Finally, we backtest our forecasted portfolio VaR using the unconditional and conditional coverage tests and independence test proposed by Kupiec (1995) and Christoffersen (1998). ${ }^{13}$

\footnotetext{
${ }^{10}$ Inclusion of the FCI makes most sense in an expanding window framework, as the FCI is quite calm in non-crises periods and volatile in crises periods. A moving window could therefore contain no crises, and hence the inclusion of the FCI results in little forecasting power.

${ }^{11}$ We do not forecast 10-step ahead at once, as the daily dynamics of the FCI fits better to an ARMA process than the 10-day dynamics.

${ }^{12} \mathrm{~A}$ second argument is the fact that if univariate returns $r_{i, t}(i=1, \ldots, J)$ are Student- $t\left(\nu_{i}\right)$ distributed, the weighted average of these $K$ return vectors may not follow a Student- $t$ distribution.

${ }^{13}$ See Appendix A for more detailed information about these tests. As indicated by Santos et al. (2013), these tests do not rank the performance of each model. Statistical tests that do take this into account are for example the CPA test of Giacomini and White (2006) or the encompassing test of Giacomini and Komunjer (2005). However, as these tests require an in-sample window of a fixed length we do not use these tests. In addition, as it is our aim to investigate whether including the FCI leads to a better coverage and/or independence a ranking of the various methods is not a necessity.
} 
We follow the suggestion of Diebold et al. (1998) by using Bonferroni bounds for the 10 sub-series in our multi-step ahead VaR-forecasts. That is, we assume that the VaR series has autocorrelation up to and including lag 9, whereas each sub-series should have correct coverage and independent VaR violations. We therefore backtest each sub-series separately with a size of $\alpha / J$. Rejecting the null hypothesis of unconditional coverage/independence occurs when the null is rejected for any of the 10 sub-series.

Tables 5 and 6 shows results of the 1-day and 10-day portfolio VaR forecasts. In the former table, we report the test results for the 1\%, $5 \%$ and $10 \%$ quantiles of the various volatility and correlation models, while the latter table shows only the tests corresponding with the $5 \%$ and $10 \%$ quantiles of the same models. In the left hand part we show the results where the quantiles are calculated using empirical quantiles, and in the right part the results with the student-t distribution. From the table it is clear that especially at the $5 \%$ and $10 \%$ VaR forecasts, including the FCI leads to an improvement in the VaR forecasts. For example, when the empirical quantile is used for $z_{q}$, the cDCC and the Factor-SplineGARCH model both fail at the $5 \%$ VaR forecasts in the unconditional coverage test, while the cDCC-X, the DC-X and cDCC model with the FCI in the volatilities do not. This result holds also for all aforementioned models except the DC-X model when the student- $t$ distribution is used for $z_{q}$ and one takes a significance level of $10 \%$. Even the Factor-SplineGARCH model with the FCI included in the variances fails in its coverage in case of $5 \%$ VaR forecasts. It seems that including the FCI in both the volatility as well as in the correlations, i.e. the cDCC-X model, delivers the best coverage, although the DC-X model fails when one uses an Student- $t$ distribution for the $5 \%$ and $10 \%$ models. This could be due to parameter uncertainty around estimating $\nu$, or due to the fact that the standardized portfolio returns does not closely match a Student- $t$ distribution.

The influence of the FCI becomes smaller for 10-day VaR forecasts, as indicated by Table 6. This is not surprising, as forecasting the Bloomberg FCI 10-steps ahead introduces more uncertainty than forecasting the same quantity one step ahead. Moreover, the effect of FCIs could be short-lived, similar to the effect of realized measures in (multivariate) volatility models, see for example Noureldin et al. (2012). In addition, the statistical tests have less power as the number of expected violations are lower, compared to the same tests applied to the 1-day VaR forecasts. Nevertheless, the $5 \%$ and $10 \%$ VaR forecasts corresponding 
Table 5: Evaluation of 1-day portfolio Value-at-Risk forecasts

This table reports the performance of various models in forecasting the $q \%$ 1-day portfolio VaR $(q=$ $1 \%, 5 \%, 10 \%)$, using an equally weighted portfolio over the period 2001-2011. The first column denotes the possible exogenous variable included in the conditional variance specification of the individual assets (market factor and ideosyncrasies). Corr.Mod represents the used correlation model. $V$ (perc) denotes the number of violations (percentage w.r.t. the total number of forecasts). FSG-cDCC is the abbreviation of the Factor-Spline-GARCH model of 2.2. $p_{u c}, p_{\text {ind }}$ and $p_{c c}$ are p-values of the (un)conditional coverage test and the independence test of Christoffersen (1998). The number of forecasted VaRs is equal to 3,025.

\begin{tabular}{|c|c|c|c|c|c|c|c|c|c|}
\hline Vol. & Corr. Mod & $\mathrm{V}($ perc $)$ & $p_{u c}$ & $p_{\text {ind }}$ & $p_{c c}$ & $\mathrm{~V}($ perc $)$ & $p_{u c}$ & $p_{\text {ind }}$ & $p_{c c}$ \\
\hline & & \multicolumn{5}{|c|}{$z_{q}$ using emp. quantile } & \multirow{2}{*}{\multicolumn{3}{|c|}{$q=t_{\hat{\nu}_{P}}^{-1}(q)$}} \\
\hline & & \multicolumn{5}{|c|}{$q=1 \%$} & & & \\
\hline - & $\mathrm{cDCC}$ & $34(1.12)$ & 0.502 & 0.379 & 0.542 & $34(1.12)$ & 0.502 & 0.399 & 0.559 \\
\hline FCI & $\mathrm{cDCC}$ & 33 (1.09) & 0.620 & 0.393 & 0.615 & $30(0.99)$ & 0.964 & 0.438 & 0.740 \\
\hline FCI & cDCC-X & 33 (1.09) & 0.620 & 0.393 & 0.615 & 33 (1.09) & 0.620 & 0.393 & 0.615 \\
\hline FCI & DC-X & $35(1.16)$ & 0.397 & 0.365 & 0.464 & $35(1.16)$ & 0.397 & 0.365 & 0.464 \\
\hline - & FSG-cDCC & $32(1.06)$ & 0.751 & 0.351 & 0.615 & $32(1.06)$ & 0.751 & 0.351 & 0.615 \\
\hline \multirow[t]{2}{*}{ FCI } & FSG-cDCC & $32(1.06)$ & 0.751 & 0.408 & 0.675 & $32(1.06)$ & 0.751 & 0.351 & 0.615 \\
\hline & & \multicolumn{8}{|c|}{$q=5 \%$} \\
\hline - & cDCC & $187(6.18)$ & 0.004 & 0.892 & 0.016 & $172(5.69)$ & 0.090 & 0.788 & 0.229 \\
\hline FCI & $\mathrm{cDCC}$ & $167(5.52)$ & 0.196 & 0.789 & 0.418 & $161(5.32)$ & 0.421 & 0.615 & 0.637 \\
\hline FCI & cDCC-X & $164(5.42)$ & 0.294 & 0.700 & 0.535 & $169(5.59)$ & 0.146 & 0.850 & 0.341 \\
\hline FCI & DC-X & $170(5.62)$ & 0.125 & 0.628 & 0.274 & 179 (5.92) & 0.024 & 0.653 & 0.071 \\
\hline 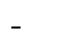 & FSG-cDCC & $197(6.51)$ & 0.000 & 0.731 & 0.001 & $181(5.98)$ & 0.016 & 0.785 & 0.053 \\
\hline \multirow[t]{2}{*}{ FCI } & FSG-cDCC & $177(5.85)$ & 0.036 & 0.834 & 0.109 & $161(5.32)$ & 0.421 & 0.615 & 0.637 \\
\hline & & \multicolumn{8}{|c|}{$q=10 \%$} \\
\hline- & cDCC & 330 (10.91) & 0.100 & 0.446 & 0.193 & $331(10.94)$ & 0.088 & 0.423 & 0.170 \\
\hline FCI & $\mathrm{cDCC}$ & $326(10.78)$ & 0.159 & 0.872 & 0.366 & $319(10.55)$ & 0.321 & 0.654 & 0.553 \\
\hline FCI & cDCC-X & $319(10.55)$ & 0.321 & 0.946 & 0.610 & $323(10.68)$ & 0.219 & 0.637 & 0.420 \\
\hline FCI & DC-X & $329(10.88)$ & 0.113 & 0.969 & 0.284 & $340(11.24)$ & 0.026 & 0.748 & 0.078 \\
\hline - & FSG-cDCC & 361 (11.93) & 0.001 & 0.371 & 0.002 & 354 (11.70) & 0.002 & 0.247 & 0.005 \\
\hline FCI & FSG-cDCC & 329 (10.88) & 0.113 & 0.360 & 0.187 & $328(10.84)$ & 0.127 & 0.381 & 0.212 \\
\hline
\end{tabular}

with the Factor-Spline-GARCH model fails for the coverage test at the $1 \%$ level when using the empirical distribution for $z_{q}$. Even if one uses the Student- $t$ distribution, the null of correct coverage is rejected at a $5 \%$ level. At the same time, cDCC model is not statistically outperformed, although it seems that the independence is not always strong, as indicated by the top-left and bottom-right part of the table ( $p$-values of 0.025 and 0.021 respectively).

All in all, we conclude that including the FCI into volatility and correlation modeling improves the VaR forecasts in case of unconditional coverage, especially at the short horizon. For the 10-day horizon, the evidence is less strong, although the Factor-Spline-GARCH model is still outperformed by the same model extended with the FCI. 
Table 6: Evaluation of 10-day portfolio Value-at-Risk forecasts

This table reports the performance of various models in forecasting the $q \%$ 10-day portfolio VaR $(q=$ $5 \%, 10 \%$ ), using an equally weighted portfolio over the period 2001-2011. The first column denotes the possible exogenous variable included in the conditional variance specification of the individual assets (market factor and ideosyncrasies). Corr.Mod. represents the used correlation model. $V_{a v}($ av.perc) denotes the average number of violations (average percentage w.r.t. the total number of VaR forecasts) over the 10 porfolios. FSG-cDCC is the abbreviation of the Factor-Spline-GARCH model of 2.2. $p_{m, u c}, p_{m, \text { ind }}$ and $p_{m, c c}$ are the minimum p-values out of the 10 tests on (un)conditional coverage test and independence test of Christoffersen (1998). The number of forecasted VaRs is equal to 303.

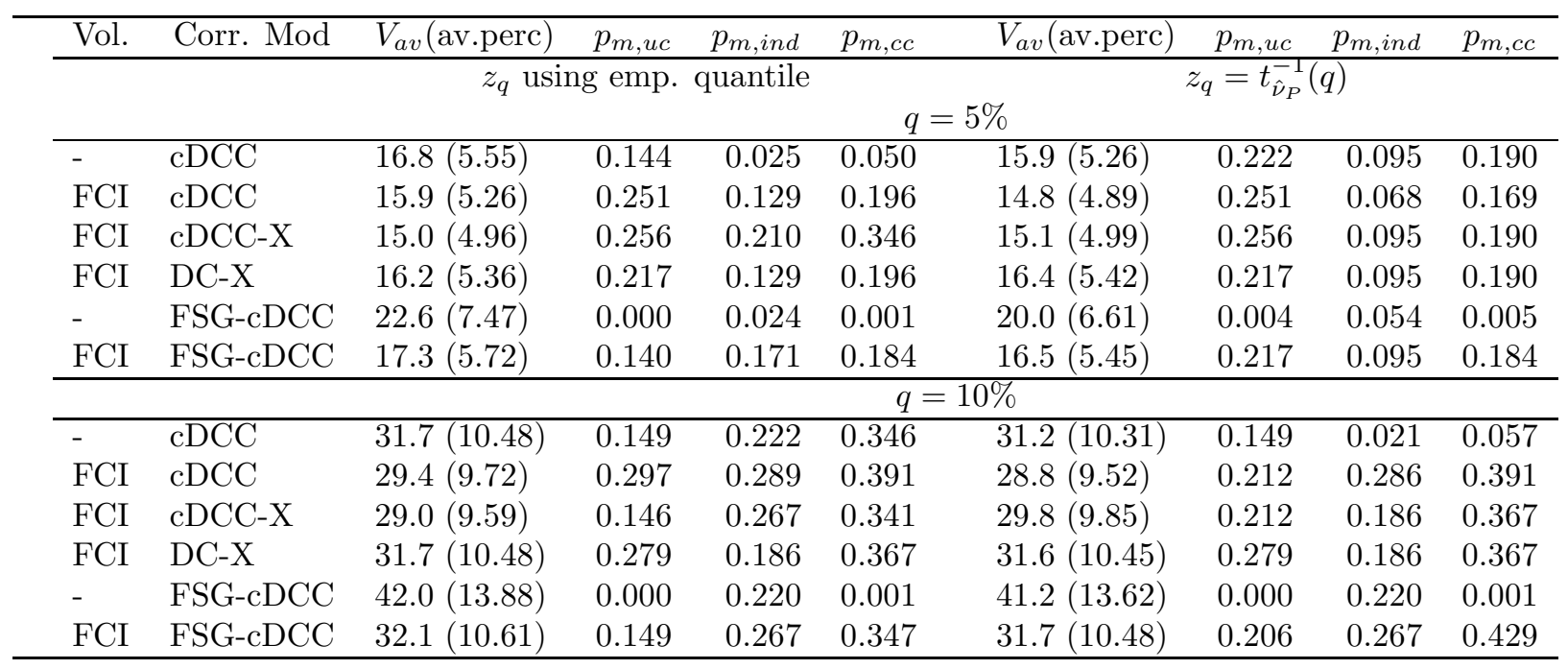




\section{The role of the VIX in the FCI}

This paper shows that including the FCI into volatility and correlation models gives a better in-sample fit as well as better VaR forecasts. The FCI is composed of various components, including money market spreads, bond market spreads and S\&P500 share prices and the VIX index (see Table 2). The question arises which of these elements drives our results. As shown in the literature, e.g. Koopman et al. (2005), the VIX is a powerful predictor of future volatility. To examine to which extent this measure drives the results on the FCI index and to what level the other components of the Bloomberg FCI have explaining/forecasting power beyond the VIX, we re-consider our in-sample and VaR analyses, now including both the FCI and VIX index.

First, we rewrite the Spline-GARCH specification in our two modeling approaches. We change the $\tau_{t}$ component (omitting the subscript $i$ ) as follows to include also the VIX index:

$$
\tau_{t}=\exp \left(\kappa_{0}+\kappa_{1} F C I_{t-1}+\kappa_{2} V I X_{t-1}\right)
$$

Note that the entire specification of the conditional variance of both model approaches is given in (3) and (9)-(12). If the significance of the $F C I_{t-1}$ vanishes, then the remaining components of the Bloomberg FCI do not have impact on the volatilities of the excess bank returns, market factor or ideosyncratic bank returns. Table 7 reports estimation results of the model parameters corresponding with Morgan Stanley, Citigroup and the market factor. In the Spline-GARCH specifications the $F C I$ variable is significant for both financial institutions. In addition, the FCI has also statistical impact on the market volatility beyond the VIX. Using the Factor-Spline-GARCH specification, the VIX seems to take away the significance in case of Citigroup. ${ }^{14}$ Nevertheless, as the FCI is significant for the market factor and Morgan Stanley, we conclude the FCI contains information beyond the VIX that impacts the volatility of asset returns. Finally, we analyse the possible influence of the VIX on the correlation between our deposit banks. The results (not shown here) suggest that the VIX does not have statistical influence on the correlations between the banks. Hence the other components of the Bloomberg FCI are additional drivers of our main result that financial conditions have impact on correlations.

\footnotetext{
${ }^{14}$ With regards to the other financial institutions of our study, the result is qualitatively similar.
} 
Table 7: In-sample estimation results with the VIX included

This table reports the estimation results of the (Factor-) Spline-GARCH-X model:

$$
\begin{aligned}
h_{t} & =g_{t} \tau_{t}, \\
g_{t} & =(1-\alpha-\delta-\gamma / 2)+\alpha \frac{\left(r_{t-1}-E_{t-1}\left(r_{t-1}\right)\right)^{2}}{\tau_{t-1}}+\gamma \frac{\left(r_{t-1}-E_{t-1}\left(r_{t-1}\right)\right)^{2}}{\tau_{t-1}} I\left[\left(r_{t-1}-E_{t-1}\left(r_{t-1}\right)\right)<0\right]+\delta g_{t-1}, \\
\tau_{t} & =\exp \left(\kappa_{0}+\kappa_{1} F C I_{t-1}+\kappa_{2} V I X_{t-1}\right),
\end{aligned}
$$

with $r_{t}$ the excess daily return or the ideosyncratic return of Morgan Stanley and Citigroup or the S\&P 500 market return $r_{m}$. Further, $E_{t-1}$ denotes the conditional expectation using all information up to and including time $t-1, F C I_{t}$ represents the Bloomberg FCI, VIX $X_{t}$ is the VIX at time $t$. The table shows Maximum Likelihood estimates, assuming a conditional Student- $t$ distribution for the standardized returns $\left(r_{t}-E_{t-1}\left(r_{t}\right) / \sqrt{h_{t}}\right.$. F-Spline-GARCH denotes the Factor-Spline-GARCH model. Standard errors are in parentheses. The sample goes from November 11, 1994, through December 30, 2011 (4,312 observations).

\begin{tabular}{lrrrrr}
\hline & \multicolumn{2}{c}{ Morgan Stanley } & \multicolumn{2}{c}{ Citigroup } & Market factor \\
\hline Coeff. & Spline-GARCH & \multicolumn{1}{c}{ F-Spline-GARCH } & Spline-GARCH & F-Spline-GARCH & \multicolumn{1}{c}{ S\&P 500 ret. } \\
\hline$\alpha$ & $0.029(0.009)$ & $0.015(0.004)$ & $0.023(0.006)$ & $0.054(0.013)$ & $0.000(0.014)$ \\
$\beta$ & $0.940(0.019)$ & $0.977(0.005)$ & $0.965(0.008)$ & $0.929(0.014)$ & $0.917(0.016)$ \\
$\delta$ & $0.029(0.013)$ & $0.011(0.007)$ & $0.020(0.008)$ & $0.024(0.012)$ & $0.077(0.020)$ \\
$\kappa_{0}$ & $0.632(0.286)$ & $0.682(0.323)$ & $0.759(0.376)$ & $0.427(0.415)$ & $-1.590(0.195)$ \\
$\kappa_{1}$ & $-0.172(0.061)$ & $-0.257(0.060)$ & $-0.093(0.065)$ & $-0.196(0.077)$ & $-0.092(0.049)$ \\
$\kappa_{2}$ & $0.551(0.113)$ & $0.285(0.091)$ & $0.519(0.103)$ & $0.402(0.109)$ & $0.707(0.093)$ \\
$\nu$ & $7.600(0.811)$ & $5.953(0.466)$ & $7.153(0.646)$ & $6.134(0.495)$ & $10.611(1.620)$ \\
\hline LogL & -9865 & -8322 & -9200 & -7709 & -6096 \\
\hline
\end{tabular}

Second, we perform VaR forecasts in the same spirit as the previous section. We consider the Factor-Spline-GARCH model, the cDCC model and the DC-X model with only the VIX included in the conditional variance specification and investigate whether similar forecast results are made as the models that contains the Bloomberg FCI in the corresponding specification. Table 8 shows that including only the VIX does not lead to correct coverage. Regarding the 1-day 5\% and 10\% VaR, the cDCC-X, DC-X and Factor-Spline-GARCH model, all significantly overestimate the number of violations. Only the cDCC model with the VIX does not fail when a Student- $t$ distribution is used. Inspecting the 10-day VaR leads to the finding that all tests perform well, except the Factor-Spline-GARCH model. The minimum $p$-value takes the value 0.009 for the $5 \%$ VaR forecasts, which is below the Bonferroni bound of size 0.01 . Hence, we reject the null hypotheses of unconditional coverage at the $10 \%$ significance level. We conclude that the other components of the FCI also have a significant impact on the VaR forecasts, in particular at the short-horizon. 


\section{Table 8: Evaluation of 1- and 10-day portfolio Value-at-Risk forecasts with the VIX index}

This table reports the performance of various models in forecasting the $q \%$ 1- and 10-day portfolio VaR ( $q=1 \%, 5 \%, 10 \%$ for 1-day VaRs and $q=5 \%, 10 \%$ for 10-day VaRs), using an equally weighted portfolio over the period 2001-2011. All models contain the VIX index in its conditional variance specification. $V$ (perc) denotes the number of violations (percentage w.r.t. the number of forecasts), $V_{a v}(a v . p e r c)$ is the average number of violations (average percentage w.r.t. the total number of VaR forecasts) over the 10 porfolios. FSG-cDCC is the abbreviation of the Factor-Spline-GARCH model of 2.2. $p_{u c}, p_{\text {ind }}$ and $p_{c c}$ are p-values of the (un)conditional coverage test and the independence test of Christoffersen (1998) in case of 1-day VaR, while $p_{m, u c}, p_{m, \text { ind }}$ and $p_{m, c c}$ are the minimum p-values of the same test over the 10 portfolios in case of the 10-day VaR forecasts. The number of forecasted VaRs is equal to 3025 (1-day) and 303 (10-day) respectively.

\begin{tabular}{|c|c|c|c|c|c|c|c|c|}
\hline Model & $\mathrm{V}($ perc $)$ & $p_{u c}$ & $p_{\text {ind }}$ & $p_{c c}$ & $\mathrm{~V}($ perc $)$ & $p_{u c}$ & $p_{\text {ind }}$ & $p_{c c}$ \\
\hline & \multicolumn{8}{|c|}{ 1-day VaR forecasts } \\
\hline & \multicolumn{4}{|c|}{$z_{q}$ using emp. quantile } & \multicolumn{2}{|c|}{$z_{q}=t_{\hat{\nu}_{P}}^{-1}(q)$} & & \\
\hline & \multicolumn{8}{|c|}{$q=1 \%$} \\
\hline $\mathrm{cDCC}$ & $31(1.02)$ & 0.891 & 0.327 & 0.613 & $30(0.99)$ & 0.964 & 0.305 & 0.590 \\
\hline $\mathrm{cDCC}-\mathrm{X}$ & $33(1.09)$ & 0.620 & 0.375 & 0.596 & $34(1.12)$ & 0.502 & 0.399 & 0.559 \\
\hline DC-X & $38(1.26)$ & 0.173 & 0.503 & 0.316 & $41(1.36)$ & 0.062 & 0.586 & 0.152 \\
\hline \multirow[t]{2}{*}{ FSG-cDCC } & $33(1.09)$ & 0.620 & 0.375 & 0.596 & $30(0.99)$ & 0.964 & 0.305 & 0.590 \\
\hline & \multicolumn{8}{|c|}{$q=5 \%$} \\
\hline $\mathrm{cDCC}$ & $179(5.92)$ & 0.024 & 0.653 & 0.071 & $168(5.55)$ & 0.169 & 0.573 & 0.332 \\
\hline $\mathrm{cDCC}-\mathrm{X}$ & $175(5.79)$ & 0.053 & 0.543 & 0.128 & $171(5.65)$ & 0.106 & 0.911 & 0.270 \\
\hline DC-X & $188(6.21)$ & 0.003 & 0.829 & 0.012 & $191(6.31)$ & 0.001 & 0.984 & 0.006 \\
\hline \multirow[t]{2}{*}{ FSG-cDCC } & $212(7.01)$ & 0.000 & 0.969 & 0.000 & $194(6.41)$ & 0.001 & 0.656 & 0.003 \\
\hline & \multicolumn{8}{|c|}{$q=10 \%$} \\
\hline $\mathrm{cDCC}$ & $334(11.04)$ & 0.060 & 0.465 & 0.131 & $327(10.81)$ & 0.142 & 0.403 & 0.240 \\
\hline $\mathrm{cDCC}-\mathrm{X}$ & $339(11.21)$ & 0.030 & 0.579 & 0.081 & $340(11.24)$ & 0.026 & 0.552 & 0.069 \\
\hline DC-X & $352(11.64)$ & 0.003 & 0.477 & 0.011 & $368(12.17)$ & 0.000 & 0.409 & 0.000 \\
\hline \multirow[t]{4}{*}{ FSG-cDCC } & $366(12.10)$ & 0.000 & 0.146 & 0.000 & $353(11.67)$ & 0.003 & 0.264 & 0.006 \\
\hline & \multicolumn{8}{|c|}{ 10-day VaR forecasts } \\
\hline & $V_{a v}($ av.perc $)$ & $p_{m, u c}$ & $p_{m, \text { ind }}$ & $p_{m, c c}$ & $V_{a v}($ av.perc $)$ & $p_{m, u c}$ & $p_{m, \text { ind }}$ & $p_{m, c c}$ \\
\hline & \multicolumn{8}{|c|}{$q=5 \%$} \\
\hline $\mathrm{cDCC}$ & $16.3(5.39)$ & 0.140 & 0.154 & 0.160 & $15.4(5.09)$ & 0.251 & 0.129 & 0.196 \\
\hline $\mathrm{cDCC}-\mathrm{X}$ & $16.5(5.45)$ & 0.144 & 0.180 & 0.163 & $14.7(4.86)$ & 0.256 & 0.068 & 0.169 \\
\hline DC-X & $17.9(5.92)$ & 0.090 & 0.077 & 0.050 & $17.1(5.65)$ & 0.144 & 0.130 & 0.163 \\
\hline \multirow[t]{2}{*}{ FSG-cDCC } & $20.2(6.68)$ & 0.009 & 0.046 & 0.013 & $18.8(6.21)$ & 0.030 & 0.054 & 0.054 \\
\hline & \multicolumn{8}{|c|}{$q=10 \%$} \\
\hline $\mathrm{cDCC}$ & $30.8(10.18)$ & 0.213 & 0.027 & 0.060 & $30.3(10.02)$ & 0.288 & 0.027 & 0.060 \\
\hline cDCC-X & $30.9(10.21)$ & 0.288 & 0.027 & 0.060 & $28.4(9.39)$ & 0.146 & 0.289 & 0.286 \\
\hline DC-X & $33.6(11.11)$ & 0.105 & 0.308 & 0.268 & $33.0(10.91)$ & 0.206 & 0.434 & 0.346 \\
\hline FSG-cDCC & $36.9(12.20)$ & 0.013 & 0.077 & 0.037 & $36.0(11.90)$ & 0.032 & 0.150 & 0.093 \\
\hline
\end{tabular}




\section{Conclusion}

We study the impact of Financial Conditions Indexes (FCIs) on volatilities and correlations of equity returns. We propose extensions of (factor-)GARCH models for volatility and DCC models for correlation that incorporate an index to measure financial conditions. In our empirical application, we use daily excess stock returns of US deposit banks during the period 1994-2011 and proxy the financial conditions by the Bloomberg FCI. This daily index summarizes the money, bond and equity market.

Our results show that financial conditions affect both the volatilities and the correlations of large US bank holding companies. Specifically, worse financial conditions are associated with higher volatilities and higher correlations. This result is both statistically and economically significant. During crisis periods, the inclusion of the FCI results in an increase in the estimated correlation of 0.15 . A forecasting exercise shows the economic gain of including the Bloomberg FCI into our volatility and correlation models. Specifically, we consider portfolio VaR forecasts using an equal-weighted portfolio and conduct 1- and 10-day ahead VaRs at various quantiles. We find that including the FCI in both the volatility and correlation specification improves the VaR forecasts at the short horizon, such that less violations are made and hence the unconditional coverage match more closely to the nominal quantile. Our results imply that risk managers and portfolio managers of financial institutions should take into account financial conditions as a predictor of future volatility and correlations.

\section{References}

Aelli, G.P. (2013), Dynamic conditional correlation: On properties and estimation, Journal of Business and Economic Statistics 31, 282-299.

Andersen, T.G., T. Bollerslev, F.X. Diebold and C. Vega (2003), Micro effects of macro announcements: Real-time price discovery in foreign exchange, American Economic Review 93, 38-62.

Andersen, T.G., T. Bollerslev, F.X. Diebold and C. Vega (2007), Real-time price discovery in global stock, bond and foreign exchange markets, Journal of International Economics $73,251-277$. 
Ang, A. and J. Chen (2002), Asymmetric correlations of equity portfolios, Journal of Financial Economics 63, 443-494.

Baele, L., G. Bekaert and K. Inghelbrecht (2010), The determinants of stock and bond return comovements, Review of Financial Studies 23, 2374-2428.

Boudt, K., J. Danielsson, S.J. Koopman and A. Lucas (2012), Regime switches in volatility and correlation of financial institutions, Working Paper.

Bracker, K. and P. Koch (1999), Economic determinants of the correlation structure across international equity markets, Journal of Economics and Business 51, 443-471.

Cappiello, L., R.F. Engle and K. Sheppard (2006), Asymmetric dynamics in the correlations of global equity and bond returns, Journal of Financial Econometrics 4, 537-572.

Christiansen, C. and A. Ranaldo (2007), Realized bond stock correlation: Macroeconomic announcement effects, Journal of Futures Markets 27, 439-469.

Christiansen, C., M. Schmeling and A. Schrimpf (2012), A comprehensive look at financial volatility prediction by economic variables, Journal of Applied Econometrics 27, 956-977.

Christoffersen, P. (1998), Evaluating interval forecasts, International Economic Review 39, $841-862$.

Diebold, F.X., T.A. Gunther and A.S. Tay (1998), Evaluating density forecasts with applications to financial risk management, International Economic Review 39, 863-883.

Engle, R.F. (2002), Dynamic conditional correlation - A simple class of multivariate GARCH models, Journal of Business and Economic Statistics 20, 339-350.

Engle, R.F. and J. Rangel (2008), The Spline-GARCH model for low-frequency volatility and its global macroeconomic causes, Review of Financial Studies 21, 1188-1222.

Engle, R.F. and K. Sheppard (2005), Evaluating the specifications of covariance models for large portfolios, Working Paper.

Engle, R.F., N. Shephard and K. Sheppard (2008), Fitting vast dimensional time-varying covariance models, Working Paper. 
Giacomini, R. and H. White (2006), Tests of conditional predictive ability, Econometrica $74,1545-1578$.

Giacomini, R. and I. Komunjer (2005), Evaluation and combination of conditional quantile forecasts, Journal of Business and Economic Statistics 23, 416-431.

Glosten, L., R. Jagannathan and D. Runkle (1993), On the relationship between the expected value and the volatility of the nominal excess return on stocks, Journal of Finance 48, 1779-1801.

Goodhart, C. and B. Hofmann (2008), Asset prices, financial conditions, and the transmission of monetary policy, Working Paper.

Guichard, S. and D. Turner (2008), Quantifying the effect of financial conditions on US activity, Working Paper.

Hafner, C. and P.H. Franses (2009), A generalized dynamic conditional correlation model: Simulation and application to many assets, Econometric Reviews 28, 612-631.

Hamilton, J.D. and G. Lin (1996), Stock market volatility and the business cycle, Journal of Applied Econometrics 11, 573-593.

Hatzius, J., P. Hooper, F.S. Mishkin, K.L. Schoenholtz and M. Watson (2010), Financial conditions indexes: A fresh look after the financial crisis, U.S. Monetary Policy Forum.

Koopman, S.J., B. Jungbacker and E. Hol (2005), Forecasting daily variability of the S\&P 100 stock index using historical, realised and implied volatility measurements, Journal of Empirical Finance 12, 445-475.

Kupiec, P.H. (1995), Techniques for verifying the accuracy of risk measurement models, Journal of Derivatives 3, 73-82.

Longin, F. and B. Solnik (2001), Extreme correlations of international equity markets, Journal of Finance 56, 676-649.

Morgan, JP (1994), Riskmetrics, Second Edition, J.P. Morgan. 
Noureldin, D., N. Shephard and K. Sheppard (2012), Multivariate high-frequency-based volatility (HEAVY) models, Journal of Applied Econometrics 27, 907-933.

Pastor, L. and P. Veronesi (2008), Learning in financial markets, Working Paper.

Paye, B. (2012), Déjà vol: Predictive regressions for aggregate stock market volatility using macroeconomic variables, Journal of Financial Economics 106, 527-546.

Perez-Quiros, G. and A. Timmermann (2001), Busines cycle asymmetries in stock returns: Evidence from higher order moments and conditional densities, Journal of Econometrics 103, 259-306.

Rangel, J.G. and R.F. Engle (2012), The factor-spline-GARCH model for high and low frequency correlations, Journal of Business and Economic Statistics 30, 109-124.

Rosenberg, M. (2009), Financial Conditions Watch, Bloomberg.

Ross, S. (1976), The arbitrage theory of capital asset pricing, Journal of Economic Theory 13, 341-360.

Santos, A.A.P., F.J. Nogales and E. Ruiz (2013), Comparing univariate and multivariate models to forecast portfolio Value-at-Risk, Journal of Financial Econometrics 11, 400441.

Schwert, G.W. (1989), Why does stock market volatility change over time?, Journal of Finance 44, 1115-1153.

Sheppard, K. (2008), Economic factors and the covariance of equity returns, Working Paper. van Dijk, D., H. Munandar and C. Hafner (2011), The euro introduction and noneuro currencies, Applied Financial Economics 21, 95-116. 


\section{A VaR backtests}

\section{A.1 The Unconditional Coverage test}

The aim of the unconditional coverage test is to investigate whether the fraction of observations inside the (estimated/predicted) interval is equal to the nominal coverage probability. Given $\tau$ observations, define $I_{t}, t=1, \ldots, \tau$ as an indicator function which takes the value 1 if the VaR lies outside the forecasted interval and 0 otherwise. Christoffersen (1998) and citetK1995 propose the following null hypothesis about correct unconditional coverage:

$$
H_{0}: \operatorname{Pr}\left[I_{t+1}=1\right]=q
$$

where $q$ is the nominal coverage probability. This probability is equal to the expectation of the indicator function $I_{t}$. Assuming independence of $I_{1}, \ldots, I_{\tau}$, the likelihood function for interval forecasts with coverage probability $p=\operatorname{Pr}\left[I_{t+1}=1\right]$ is given by

$$
L\left(p ; I_{\tau}, I_{\tau-1}, \ldots, I_{1}\right)=p^{T_{1}}(1-p)^{T_{0}}
$$

where $T_{1}$ and $T_{0}$ represent the amount of violations and non-violations respectively. The likelihood under the null hypothesis given above with $p=q$ is compared with the likelihood under the alternative hypothesis where $p$ equals the failure rate $f$. This rate is estimated by $\hat{f}=\widehat{\operatorname{Pr}}\left(I_{t+1}=1\right)=T_{1} /\left(T_{0}+T_{1}\right)$. The LR statistic is then given by

$$
L R_{u c}=-2 \log \left(\frac{q^{M}(1-q)^{T-M}}{\hat{f}^{M}(1-\hat{f})^{T-M}}\right)
$$

and is asymptotically distributed as a $\chi^{2}$ distribution with one degrees of freedom.

\section{A.2 The Independence test}

The independence test advocated by Christoffersen (1998) investigates whether the occurrences violations are spread out over the sample instead of appearing in clusters. This 'independence' is tested against the specific alternative of a first-order Markov chain. Using 
the same notation as in the previous section, this boils down to

$$
H_{0}: \operatorname{Pr}\left[I_{t+1}=1 \mid I_{t}\right]=\operatorname{Pr}\left[I_{t+1}\right] \quad t=1, \ldots, \tau .
$$

If $I_{t+1}$ follows a first-order Markov chain with transition probability matrix, then

$$
\Pi_{1}=\left(\begin{array}{cc}
1-\pi_{01} & \pi_{01} \\
1-\pi_{11} & \pi_{11}
\end{array}\right)
$$

where $\pi_{i j}=\operatorname{Pr}\left[I_{t+1}=j \mid I_{t}=i\right]$. The likelihood is equal to

$$
L\left(\Pi_{1} ; I_{\tau}, I_{\tau-1}, \ldots, I_{1}\right)=\left(1-\pi_{01}\right)^{T_{00}} \pi_{01}^{T_{01}}\left(1-\pi_{11}\right)^{T_{10}} \pi_{11}^{T_{11}},
$$

where $T_{i j}$ is equal to the number of observations out of $(\tau-1)$ such that $I_{t+1}=j$ and $I_{t}=i$. The maximum likelihood estimate of $\Pi_{1}$ is given by

$$
\hat{\Pi}_{1}=\left(\begin{array}{cc}
\frac{T_{00}}{T_{00}+T_{01}} & \frac{T_{01}}{T_{00}+T_{01}} \\
\frac{T_{10}}{T_{10}+T_{11}} & \frac{T_{11}}{T_{10}+T_{11}}
\end{array}\right) .
$$

Under the null hypothesis of independence, $\pi_{01}=\pi_{11} \equiv \pi_{2}$, which leads to the corresponding likelihood function

$$
L\left(\pi_{2} ; I_{\tau}, I_{\tau-1}, \ldots, I_{1}\right)=\left(1-\pi_{2}\right)^{T_{00}+T_{01}} \pi_{2}^{T_{01}+T_{11}} .
$$

Then by estimating $\pi_{2}$ with $\hat{\pi}_{2}=\left(T_{00}+T_{01}\right) /\left(T_{00}+T_{01}+T_{10}+T_{11}\right)$ the likelihood ratio test of independence is computed as

$$
L R_{u c}=-2 \log \left(\frac{L\left(\hat{\pi}_{2} ; I_{\tau}, I_{\tau-1}, \ldots, I_{1}\right)}{L\left(\hat{\Pi}_{1} ; I_{\tau}, I_{\tau-1}, \ldots, I_{1}\right)}\right) \stackrel{a s y}{\rightarrow} \chi^{2}
$$

under the null hypothesis. Finally, as shown by Christoffersen (1998), the unconditional coverage and the independence test can be combined to test correct conditional coverage by adding the two likelihood ratio test statistics. Under $H_{0}$ this statistic follows asymptotically a $\chi^{2}$ distribution with two degrees of freedom. 


\section{B In-sample Volatilities - All assets}

Table A.1: In-sample estimation results - Spline-GARCH Models - All assets This table reports the estimation results of the Spline-GARCH(-X) model:

$$
\begin{aligned}
& r_{i, t}=\mu_{i}+\sqrt{g_{i, t} \tau_{i, t}} \varepsilon_{i, t}, \\
& g_{i, t}=\left(1-\alpha_{i}-\delta_{i}-\gamma_{i} / 2\right)+\alpha_{i} \frac{\left(r_{i, t-1}-\mu_{i}\right)^{2}}{\tau_{i, t-1}}+\gamma_{i} \frac{\left(r_{i, t-1}-\mu_{i}\right)^{2}}{\tau_{i, t-1}} I\left[\left(r_{i, t-1}-\mu_{i}\right)<0\right]+\delta_{i} g_{i, t-1}, \\
& \tau_{i, t}=\exp \left(\kappa_{i, 0}+\kappa_{i, 1} F C I_{t-1}\right),
\end{aligned}
$$

with $r_{i, t}$ the daily excess return of holding bank $i,(i=1, \ldots, 16), F C I_{t}$ represents the Bloomberg FCI. Panel A of the table shows Maximum Likelihood estimates of the Spline-GARCH model without any added exogenous variable, assuming a conditional Student- $t$ distribution for $\varepsilon_{t}$. Panel B shows parameter estimates of the Spline-GARCH-X model with the Bloomberg FCI included as exogenous variable. $\mathrm{Nr}$ in the first column corresponds with the order of Table 1. Standard errors are in parentheses. The sample goes from November 11, 1994, through December 30, 2011 (4,312 observations) for all numbers except nr. 3 and 7

\begin{tabular}{|c|c|c|c|c|c|c|c|}
\hline \multicolumn{8}{|c|}{ Panel A: Spline-GARCH } \\
\hline Nr. & $\mu$ & $\alpha$ & $\delta$ & $\gamma$ & $\kappa_{0}$ & & $\nu$ \\
\hline 1. & $0.007(0.014)$ & $0.054(0.011)$ & $0.901(0.013)$ & $0.078(0.016)$ & $1.889(0.340)$ & & $6.405(0.517)$ \\
\hline 2. & $0.012(0.016)$ & $0.039(0.010)$ & $0.911(0.011)$ & $0.088(0.014)$ & $1.472(0.309)$ & & $6.105(0.471)$ \\
\hline 3. & $0.059(0.026)$ & $0.052(0.013)$ & $0.924(0.008)$ & $0.049(0.015)$ & $-0.414(0.027)$ & & $7.947(0.024)$ \\
\hline 4. & $0.021(0.023)$ & $0.024(0.007)$ & $0.931(0.008)$ & $0.086(0.012)$ & $2.174(0.415)$ & & $8.521(0.902)$ \\
\hline 5. & $0.032(0.022)$ & $0.024(0.007)$ & $0.933(0.008)$ & $0.080(0.012)$ & $1.990(0.334)$ & & $7.889(0.766)$ \\
\hline 6. & $0.014(0.032)$ & $0.022(0.009)$ & $0.922(0.011)$ & $0.104(0.017)$ & $2.397(0.042)$ & & $5.737(0.082)$ \\
\hline 7. & $0.012(0.060)$ & $0.062(0.016)$ & $0.883(0.017)$ & $0.084(0.025)$ & $1.504(0.345)$ & & $5.858(0.559)$ \\
\hline 8. & $0.029(0.020)$ & $0.046(0.009)$ & $0.919(0.009)$ & $0.064(0.013)$ & $1.963(0.435)$ & & $7.150(0.620)$ \\
\hline 9. & $0.014(0.017)$ & $0.037(0.010)$ & $0.920(0.013)$ & $0.079(0.016)$ & $1.987(0.413)$ & & $6.446(0.545)$ \\
\hline 10. & $0.025(0.024)$ & $0.031(0.008)$ & $0.937(0.009)$ & $0.057(0.012)$ & $1.575(0.334)$ & & $7.568(0.740)$ \\
\hline 11. & $0.037(0.018)$ & $0.044(0.010)$ & $0.919(0.010)$ & $0.068(0.013)$ & $1.528(0.363)$ & & $7.499(0.677)$ \\
\hline 12. & $0.023(0.018)$ & $0.037(0.008)$ & $0.933(0.009)$ & $0.050(0.012)$ & $1.321(0.286)$ & & $7.014(0.616)$ \\
\hline 13. & $0.029(0.027)$ & $0.025(0.007)$ & $0.923(0.009)$ & $0.094(0.014)$ & $2.368(0.309)$ & & $7.203(0.667)$ \\
\hline 14. & $0.015(0.016)$ & $0.036(0.007)$ & $0.928(0.009)$ & $0.069(0.012)$ & $2.166(0.399)$ & & $7.151(0.609)$ \\
\hline 15. & $0.017(0.014)$ & $0.028(0.010)$ & $0.922(0.012)$ & $0.095(0.014)$ & $1.961(0.437)$ & & $6.864(0.591)$ \\
\hline 16. & $0.046(0.031)$ & $0.027(0.007)$ & $0.945(0.009)$ & $0.051(0.011)$ & $2.254(0.334)$ & & $5.535(0.359)$ \\
\hline \multicolumn{8}{|c|}{ Panel B: Spline-GARCH-X } \\
\hline Nr. & $\mu$ & $\alpha$ & $\delta$ & $\bar{\gamma}$ & $\kappa_{0}$ & $\kappa_{1}$ & $\nu$ \\
\hline 1. & $0.000(0.017)$ & $0.052(0.012)$ & $0.910(0.015)$ & $0.058(0.015)$ & $1.578(0.255)$ & $-0.354(0.067)$ & $6.415(0.544)$ \\
\hline 2. & $0.006(0.015)$ & $0.037(0.010)$ & $0.917(0.012)$ & $0.073(0.013)$ & $1.275(0.242)$ & $-0.359(0.063)$ & $6.072(0.489)$ \\
\hline 3. & $0.028(0.022)$ & $0.070(0.014)$ & $0.883(0.016)$ & $0.048(0.019)$ & $1.042(0.156)$ & $-0.436(0.062)$ & $7.989(0.958)$ \\
\hline 4. & $0.014(0.031)$ & $0.020(0.006)$ & $0.944(0.008)$ & $0.069(0.011)$ & $2.009(0.392)$ & $-0.282(0.048)$ & $8.667(0.957)$ \\
\hline 5. & $0.026(0.020)$ & $0.021(0.006)$ & $0.948(0.008)$ & $0.055(0.012)$ & $1.755(0.295)$ & $-0.272(0.051)$ & $8.139(0.846)$ \\
\hline 6. & $0.006(0.012)$ & $0.021(0.011)$ & $0.936(0.020)$ & $0.072(0.020)$ & $1.926(0.362)$ & $-0.337(0.055)$ & $5.679(0.463)$ \\
\hline 7. & $0.010(0.020)$ & $0.050(0.014)$ & $0.900(0.024)$ & $0.048(0.021)$ & $1.029(0.146)$ & $-0.476(0.050)$ & $6.405(0.671)$ \\
\hline 8. & $0.024(0.020)$ & $0.041(0.009)$ & $0.937(0.010)$ & $0.039(0.012)$ & $1.635(0.376)$ & $-0.327(0.052)$ & $7.334(0.663)$ \\
\hline 9. & $0.008(0.017)$ & $0.024(0.010)$ & $0.950(0.015)$ & $0.044(0.014)$ & $1.536(0.357)$ & $-0.369(0.045)$ & $6.306(0.554)$ \\
\hline 10. & $0.021(0.029)$ & $0.024(0.008)$ & $0.958(0.009)$ & $0.030(0.010)$ & $1.293(0.264)$ & $-0.336(0.046)$ & $7.952(0.829)$ \\
\hline 11. & $0.029(0.019)$ & $0.038(0.010)$ & $0.932(0.011)$ & $0.052(0.012)$ & $1.293(0.295)$ & $-0.354(0.053)$ & $7.658(0.734)$ \\
\hline 12. & $0.019(0.019)$ & $0.029(0.008)$ & $0.952(0.009)$ & $0.029(0.010)$ & $1.089(0.237)$ & $-0.317(0.047)$ & $7.091(0.635)$ \\
\hline 13. & $0.021(0.030)$ & $0.029(0.008)$ & $0.939(0.012)$ & $0.046(0.014)$ & $1.891(0.201)$ & $-0.403(0.050)$ & $7.460(0.759)$ \\
\hline 14. & $0.006(0.033)$ & $0.031(0.007)$ & $0.948(0.010)$ & $0.038(0.014)$ & $1.846(0.035)$ & $-0.323(0.089)$ & $7.081(0.094)$ \\
\hline 15. & $0.010(0.014)$ & $0.016(0.009)$ & $0.945(0.012)$ & $0.074(0.012)$ & $1.671(0.400)$ & $-0.317(0.049)$ & $7.038(0.642)$ \\
\hline 16. & $0.035(0.027)$ & $0.014(0.005)$ & $0.972(0.006)$ & $0.026(0.007)$ & $1.880(0.319)$ & $-0.317(0.041)$ & $5.575(0.368)$ \\
\hline
\end{tabular}
(Wachovia Corp. and National City Corp.). For these two financial institutions, the sample goes from November 11, 1994, through December 30, 2008 (3,556 observations). 
Table A.2: In-sample estimation results - Factor-Spline-GARCH models - All assets

This table reports the estimation results of the Factor-Spline-GARCH model. The volatility is formulated as a Spline-GARCH-X model:

$$
\begin{aligned}
r_{i, t} & =\mu_{i, t}+\sqrt{g_{i, t} \tau_{i, t}} \varepsilon_{i, t}, \\
g_{i, t} & =\left(1-\alpha_{i}-\delta_{i}-\gamma_{i} / 2\right)+\alpha_{i} \frac{\left(r_{i, t-1}-\mu_{i, t}\right)^{2}}{\tau_{t-1}}+\gamma_{i} \frac{\left(r_{i, t-1}-\mu_{i, t}\right)^{2}}{\tau_{t-1}} I\left[\left(r_{i, t-1}-\mu_{i, t}\right)<0\right]+\delta_{i} g_{i, t-1}, \\
\tau_{i, t} & =\exp \left(\kappa_{i, 0}+\kappa_{i, 1} F C I_{t-1}\right),
\end{aligned}
$$

with $r_{i, t}$ the daily excess return of holding bank $i,(i=m, 1, \ldots, 16)$ and the S\&P 500 market return. In addition, $\mu_{i, t}=\xi_{i}+\beta_{i} r_{m, t}$ with $r_{m, t}$ the excess market return at day $t$. Otherwise, $\mu_{i, t}=\mu_{m}$ in case of the market return. $F C I_{t}$ represents the Bloomberg FCI. Panel A of the table shows Maximum Likelihood estimates of the Spline-GARCH model without any added exogenous variable, assuming a conditional Student- $t$ distribution for $\varepsilon_{t}$. Panel B shows parameter estimates of the Spline-GARCH-X model with the Bloomberg FCI included as exogenous variable. $N r$ in the first column corresponds with the order of Table 1. Standard errors are in parentheses. The sample goes from November 11, 1994, through December 30, 2011 (4,312 observations) for all numbers except nr. 3 and 7 (Wachovia Corp. and National City Corp.).

\begin{tabular}{|c|c|c|c|c|c|c|c|}
\hline \multicolumn{8}{|c|}{ Panel A: Spline-GARCH } \\
\hline Nr. & $\beta$ & $\alpha$ & $\delta$ & $\gamma$ & $\kappa_{0}$ & & $\nu$ \\
\hline \multicolumn{8}{|c|}{ Panel A } \\
\hline 1. & $0.980(0.022)$ & $0.082(0.016)$ & $0.897(0.017)$ & $0.027(0.016)$ & $1.508(0.384)$ & & $5.352(0.370)$ \\
\hline 2. & $0.844(0.018)$ & $0.053(0.012)$ & $0.910(0.012)$ & $0.065(0.014)$ & $1.303(0.378)$ & & $5.473(0.382)$ \\
\hline 3. & $0.977(0.019)$ & $0.106(0.019)$ & $0.864(0.020)$ & $0.037(0.021)$ & $1.073(0.379)$ & & $5.856(0.509)$ \\
\hline 4. & $0.957(0.018)$ & $0.051(0.010)$ & $0.929(0.010)$ & $0.037(0.013)$ & $1.828(0.509)$ & & $6.759(0.572)$ \\
\hline 5. & $1.327(0.019)$ & $0.028(0.006)$ & $0.952(0.006)$ & $0.036(0.009)$ & $1.332(0.370)$ & & $5.597(0.384)$ \\
\hline 6. & $1.241(0.020)$ & $0.043(0.012)$ & $0.923(0.014)$ & $0.063(0.016)$ & $2.035(0.462)$ & & $4.812(0.295)$ \\
\hline 7. & $0.930(0.018)$ & $0.120(0.019)$ & $0.871(0.018)$ & $0.000(0.020)$ & $1.518(0.039)$ & & $4.809(0.066)$ \\
\hline 8. & $1.011(0.019)$ & $0.058(0.011)$ & $0.924(0.011)$ & $0.032(0.013)$ & $1.579(0.493)$ & & $5.893(0.438)$ \\
\hline 9. & $1.060(0.018)$ & $0.059(0.015)$ & $0.933(0.017)$ & $0.008(0.015)$ & $1.232(0.474)$ & & $5.144(0.351)$ \\
\hline 10. & $1.045(0.018)$ & $0.045(0.010)$ & $0.944(0.010)$ & $0.018(0.012)$ & $1.158(0.415)$ & & $5.253(0.352)$ \\
\hline 11. & $1.005(0.018)$ & $0.079(0.013)$ & $0.907(0.011)$ & $0.016(0.014)$ & $1.072(0.377)$ & & $5.888(0.450)$ \\
\hline 12. & $0.995(0.017)$ & $0.066(0.014)$ & $0.920(0.015)$ & $0.016(0.013)$ & $0.833(0.346)$ & & $5.717(0.413)$ \\
\hline 13. & $1.605(0.023)$ & $0.028(0.007)$ & $0.943(0.009)$ & $0.050(0.011)$ & $1.648(0.317)$ & & $5.538(0.394)$ \\
\hline 14. & $1.301(0.020)$ & $0.065(0.012)$ & $0.914(0.012)$ & $0.036(0.014)$ & $1.588(0.447)$ & & $5.984(0.445)$ \\
\hline 15. & $0.909(0.016)$ & $0.075(0.018)$ & $0.911(0.016)$ & $0.024(0.016)$ & $1.625(0.554)$ & & $5.168(0.348)$ \\
\hline 16. & $1.310(0.031)$ & $0.033(0.005)$ & $0.967(0.003)$ & $0.000(0.007)$ & $-4.193(0.076)$ & & $4.595(0.133)$ \\
\hline \multicolumn{8}{|c|}{ Panel B: Spline-GARCH-X } \\
\hline Nr. & $\bar{\beta}$ & $\alpha$ & $\bar{\theta}$ & $\gamma$ & $\kappa_{0}$ & $\kappa_{1}$ & $\nu$ \\
\hline 1. & $0.986(0.022)$ & $0.077(0.018)$ & $0.898(0.021)$ & $0.023(0.015)$ & $1.158(0.241)$ & $-0.376(0.054)$ & $5.438(0.401)$ \\
\hline 2. & $0.844(0.018)$ & $0.041(0.012)$ & $0.924(0.013)$ & $0.059(0.012)$ & $1.086(0.316)$ & $-0.318(0.055)$ & $5.563(0.399)$ \\
\hline 3. & $0.981(0.019)$ & $0.103(0.019)$ & $0.865(0.024)$ & $0.020(0.021)$ & $0.675(0.219)$ & $-0.418(0.062)$ & $6.243(0.594)$ \\
\hline 4. & $0.961(0.018)$ & $0.033(0.009)$ & $0.946(0.010)$ & $0.041(0.011)$ & $1.632(0.445)$ & $-0.339(0.046)$ & $6.871(0.602)$ \\
\hline 5. & $1.338(0.019)$ & $0.015(0.004)$ & $0.971(0.005)$ & $0.026(0.007)$ & $1.160(0.311)$ & $-0.339(0.039)$ & $5.962(0.445)$ \\
\hline 6. & $1.246(0.020)$ & $0.032(0.013)$ & $0.934(0.018)$ & $0.061(0.016)$ & $1.746(0.482)$ & $-0.356(0.048)$ & $4.790(0.312)$ \\
\hline 7. & $0.934(0.019)$ & $0.066(0.027)$ & $0.910(0.043)$ & $0.016(0.020)$ & $0.695(0.228)$ & $-0.464(0.057)$ & $5.295(0.461)$ \\
\hline 8. & $1.013(0.019)$ & $0.048(0.010)$ & $0.939(0.010)$ & $0.023(0.011)$ & $1.277(0.418)$ & $-0.310(0.049)$ & $6.068(0.469)$ \\
\hline 9. & $1.062(0.018)$ & $0.021(0.007)$ & $0.971(0.007)$ & $0.014(0.007)$ & $1.047(0.377)$ & $-0.362(0.037)$ & $5.121(0.349)$ \\
\hline 10. & $1.047(0.018)$ & $0.034(0.009)$ & $0.959(0.010)$ & $0.010(0.010)$ & $0.940(0.338)$ & $-0.320(0.047)$ & $5.500(0.386)$ \\
\hline 11. & $1.008(0.018)$ & $0.062(0.013)$ & $0.918(0.012)$ & $0.025(0.014)$ & $0.806(0.301)$ & $-0.352(0.042)$ & $6.188(0.510)$ \\
\hline 12. & $0.998(0.017)$ & $0.043(0.015)$ & $0.944(0.017)$ & $0.017(0.009)$ & $0.569(0.286)$ & $-0.315(0.049)$ & $5.846(0.434)$ \\
\hline 13 & $1.612(0.023)$ & $0.018(0.005)$ & $0.976(0.007)$ & $0.010(0.009)$ & $1.201(0.292)$ & $-0.401(0.040)$ & $5.921(0.457)$ \\
\hline 14 & $1.306(0.020)$ & $0.054(0.012)$ & $0.929(0.013)$ & $0.026(0.012)$ & $1.240(0.369)$ & $-0.401(0.053)$ & $6.150(0.484)$ \\
\hline 15. & $0.911(0.016)$ & $0.062(0.020)$ & $0.919(0.019)$ & $0.029(0.013)$ & $1.357(0.505)$ & $-0.322(0.058)$ & $5.210(0.366)$ \\
\hline 16. & $1.383(0.027)$ & $0.017(0.005)$ & $0.973(0.007)$ & $0.017(0.006)$ & $1.589(0.330)$ & $-0.289(0.038)$ & $4.444(0.237)$ \\
\hline
\end{tabular}
For these two financial institutions, the sample goes from November 11, 1994, through December 30, 2008 (3,556 observations). 